\title{
COMO AS ABELHAS PERCEBEM AS FLORES E POR QUE ISTO É IMPORTANTE?
}

\author{
Lílian Rodrigues Ferreira de Melo ${ }^{1 *}$, Bárbara Matos da Cunha Guimarães ${ }^{1}$, \\ Gudryan Jackson Barônio ${ }^{1,2}$, Larissa Chagas de Oliveira ${ }^{3}$, Renan Kobal de Oliveira \\ Alves Cardoso ${ }^{4}$, Thayane Nogueira Araújo ${ }^{1}$ \& Francismeire Jane Telles ${ }^{1}$
}

1 Universidade Federal de Uberlândia, Instituto de Biologia, Programa de Pós-Graduação em Ecologia e Conservação de Recursos Naturais, Campus Umuarama, CEP 38400-902, Uberlândia, MG, Brasil.

2 Universidade Federal do ABC, Programa de Pós-Graduação com Evolução e Diversidade, Campus São Bernardo do Campo, CEP 09606-070, São Bernardo do Campo, SP, Brasil.

${ }^{3}$ Universidade Federal de Uberlândia, Instituto de Biologia, Programa de Pós-Graduação em Biologia Vegetal, Campus Umuarama, CEP 38400-902, Uberlândia, MG, Brasil.

${ }^{4}$ Universidade de São Paulo, Programa de Pós-Graduação em Entomologia, Departamento de Biologia, Av. Bandeirantes, n. 3900, Monte Alegre, CEP 14040-901, Ribeirão Preto, SP, Brasil

E-mails: lilianrferreiramelo@gmail.com (*autor correspondente); bmatoscg@gmail.com; gudryan@gmail.com; larissacholiveira@hotmail.com; renankcardoso@gmail.com; meirecuesta@gmail.com

Resumo: A cognição animal pode ser definida como a capacidade apresentada por um organismo em adquirir, reter e posteriormente utilizar a informação sensorial na tomada de decisões, em diferentes contextos. A compreensão de como os sinais emitidos no ambiente interagem com os canais sensoriais dos animais capazes de percebê-los ajuda a desvendar o significado ecológico e evolutivo das interações entre os organismos. No contexto da polinização, a atração de visitantes à flor é atribuída a uma grande diversidade de sinais, principalmente os visuais e olfativos. Além de atuarem como atrativos, esses sinais também podem exibir funções relacionadas à comunicação da presença/ausência de recursos. Não obstante, outras modalidades sensoriais menos conhecidas, também desempenham papel relevante na interação entre flores e visitantes. A ideia central do presente trabalho é expor, através de exemplos relevantes da literatura, os principais sinais emitidos pelas flores e percebidos pelas abelhas, seja através do uso de uma única modalidade sensorial ou através de múltiplas modalidades. Independente da modalidade sensorial e da complexidade dos estímulos, estudos sobre as interações entre plantas e seus visitantes florais ganham maior entendimento das relações se consideramos os diferentes aspectos relacionados com o sinal que está sendo emitido, e a direcionalidade do mesmo, ou simplesmente a capacidade ou não de ser percebido. Quantificar os processos, suas causas e consequências reforça o entendimento de padrões evolutivos, ecológicos e comportamentais entre os organismos interagentes, tanto em relações puramente mutualistas quanto em relações antagonistas.

Palavras-chave: cognição; interação planta-polinizador; polinizadores; sinais florais, sistema sensorial.

HOW DO BEES PERCEIVE FLOWERS AND WHY IT IS IMPORTANT? Animal cognition can be defined as the ability of an organism to acquire, retain and subsequently use the sensory information during decision-making processes in different contexts. The understanding of how the signals emitted in the environment interact with the sensory system of animals capable of perceiving them helps to unravel the 
ecological and evolutionary significance of the interactions between organisms. In the context of pollination, the attraction of visitors to the flower is attributed to a great diversity of signals, especially visuals and olfactory. In addition to acting as attractants, they can also exhibit functions related to the communication of the presence/absence of resources. Nevertheless, other sensory modalities, also play a relevant role on the interaction between flowers and visitors. The central idea of the present study is to present, through relevant examples from the literature, the main signals emitted by the flowers and perceived by the bees, either by a single sensory modality or through multiple modalities. Regardless of the sensory modality and the complexity of the stimuli, studies of the interactions between plants and their floral visitors can be better understood and detailed if we consider the different aspects related to the signal being emitted and the directionality of the same, or simply the capacity or not to be perceived. Quantifying processes, their causes and consequences reinforce the understanding of evolutionary, ecological and behavioral patterns among interacting organisms, both in mutualistic and antagonistic relationships.

Keywords: cognition; floral attractants; plant-pollinator interaction; pollinators; sensory systems.

\section{INTRODUÇÃO}

A relação entre plantas e seus visitantes florais gera uma série de questionamentos envolvendo interações mutualistas e antagonistas, dando lugar a estudos que colaboram para o desenvolvimento da biologia da polinização atual. Por sua natureza interdisciplinar, esses estudos abrangem diferentes perspectivas com um propósito comum: entender os aspectos fundamentais que estruturam os processos por trás das interações ecológicas entre plantas e visitantes florais. Entre algumas das abordagens mais recentes nos estudos de ecologia e evolução das interações entre flores e visitantes, estão as capacidades sensorial e cognitiva dos animais em selecionar, responder e interpretar os sinais emitidos pelas flores (Chittka \& Thomson 2001, Raguso 2004, Brito et al. 2014). Uma vez percebido, a avaliação e decodificação do sinal envolve a capacidade cognitiva do organismo receptor, além de processos mais complexos.

Cognição animal pode ser definida como a capacidade apresentada por um organismo em adquirir, processar, reter e, posteriormente, utilizar a informação captada, através dos sistemas sensoriais, no processo de tomada de decisões em diferentes contextos relevantes para o animal
(Dukas 2004, Dukas \& Ratcliffe 2009, Brito et al. 2014). Quando apenas um canal sensorial é explorado ou ativado por um sinal, este é denominado unimodal, enquanto um sinal que estimula múltiplas modalidades sensoriais é denominado multimodal. No mesmo contexto, um conjunto de sinais que estimulam um único ou vários canais sensoriais são chamados de sinais multicomponentes. Sinais multicomponentes podem ser unimodais (quando eles estimulam apenas um canal sensorial) ou multimodais (quando dois ou mais canais sensoriais ativados). Por exemplo, a coloração das pétalas e os guias de néctar atuam como diferentes componentes que estimulam apenas o canal visual - multicomponente unimodal (Leonard \& Papaj 2011), assim como os odores florais, que originalmente consistem em uma mistura de diferentes compôstos voláteis, mas que formam um sinal composto, atuando no canal olfativo (Raguso 2008).

Alguns estudos têm demonstrado que os animais diferem nas suas habilidades de discriminar, perceber e relembrar estímulos definidos através de um único componente em comparação com estímulos definidos por componentes múltiplos (veja mais em BroJørgensen 2010). Alguns sinais trabalham sinergicamente com outras modalidades no processo 
de atração e seus efeitos nos visitantes são contexto-dependentes (Raguso \& Willis 2002, Junker \& Parachnowitsch 2015). Ainda, os visitantes são capazes de aprender a reconhecer componentes considerados chave de um conjunto de sinais mais complexos, como ocorre com as fragrâncias florais, em que determinados grupos respondem exclusivamente a presença e concentrações específicas de determinados voláteis (Reinhard et al. 2010).

Independente da origem e complexidade do sinal, ao emergirem e durante o transcurso de suas vidas, os visitantes florais devem responder a uma variedade de estímulos ocorrendo simultâneamente no espaço. Respostas iniciais são moduladas por preferências inatas, seja por formas que se assemelham a morfologias florais (Lehrer et al. 1995), por cores e odores (filogeneticamente determinadas) (Lunau \& Maier 1995, Gumbert 2000, Milet-Pinheiro et al. 2012), ou ainda, através de preferências moduladas pelas condições da flora local (Chittka et al. 2004). As preferências inatas guiam o forrageio de animais recém-emergidos em direção à estímulos nunca percebidos, mas que, por razões ecológicas e evolutivas, resultam vantajosos para eles. Porém, estímulos que correspondam às preferências inatas podem nem sempre estar disponíveis na natureza no momento do surgimento dos indivíduos ou, ainda que os visitantes mostrem preferências inatas por certos estímulos, a escolha em comunidades naturais pode não refletir essas preferências (Heinrich 1979, Reverté et al. 2016). Por isso, no processo de sinalização-resposta, devem existir outros mecanismos capazes de assegurar a obtenção da informação, e consequentemente dos recursos, de maneira contínua ao longo do tempo. A modularidade gerada pela variação espacial e/ou temporal na comunidade de plantas e visitantes florais, dá lugar à processos associativos, através do aprendizado (Heinrich 1979).

O processo de associação entre os estímulos florais e os recursos disponibilizados pela planta, é o principal mecanismo de determinação da persistência das interações (Chittka \& Thomson 2001), levando, por exemplo, a uma constância na frequência das visitas (Chittka et al. 1999, Márquez
2009). A constância floral pode se dar através de relações especializadas (Praz et al. 2008, Klaus Lunau et al. 2011) ou através de especializações temporais, nas quais animais considerados generalistas se especializam temporalmente na exploração de uma ou poucas espécies de plantas capazes de suprir as necessidades relacionadas com a quantidade e/ou qualidade dos recursos procurados (Wilson \& Stine 1996, Schiestl et al. 2010, Grüter et al. 2011). Contudo, entre os mecanismos de sinalização, nem todos são considerados honestos e, dependendo do caso, o estabelecimento de uma relação mutualista pode ser afetado. No processo de sinalização honesta, um sinal é emitido e o visitante é recompensado pela visita - e no melhor dos casos pelo serviço de polinização prestado - com a obtenção do recurso procurado. No entanto, existem plantas que se beneficiam de sinais honestos emitidos ao seu redor (espécie modelo) para enganar visitantes e receber visitas sem recompensá-los, a partir do mimetismo de sinais (Ellis \& Johnson 2010, Jürgens et al. 2013). No caso dos mecanismos envolvendo decepção alimentar, nos quais ambos modelo e espécie mimética coocorrem, a percepção e recusa da sinalização desonesta é dificultada, já que naturalmente durante as visitas, os forrageadores encontram variações nas recompensas entre flores/inflorescências na população - ora com recompensa porque ainda não foi visitada, ora sem recompensa após uma visita prévia de outro indivíduo.

No contexto das comunidades, as interações flor-visitante podem resultar em processos de competição ou facilitação, tanto entre as plantas na tentativa de atrair e selecionar seus polinizadores - como entre visitantes (Mitchell et al. 2009a, Irwin et al. 2010, Bergamo et al. 2017). No entanto, na perspectiva da comunidade, onde há emissão de sinais de diferentes naturezas, intensidades e frequências, é possível que a presença de múltiplos sinais florais seja fonte de ruído no processo de percepção das flores e na associação de seus recursos pelos visitantes (Chittka et al. 1999, Chittka \& Thomson 2001, Rusch et al. 2016). Por outro lado, também podemos imaginar que a complexidade resultante da existência de variações nos atributos florais 
(e.g. cor, tamanho, forma, simetria, odor), e o fato dos visitantes exibirem certas preferências por determinados sinais, ou serem capazes de filtrar sinais mais relevantes, pode ajudar durante o processo de seleção e reconhecimento das espécies vegetais entre as várias presentes no ambiente (Lehrer et al. 1995, Gegear \& Laverty 2001, Kárpáti et al. 2013, Riffell et al. 2014).

O presente trabalho consiste em uma revisão sobre os principais sinais emitidos pelas flores no processo de atração de um grupo com destacada relevância nos estudos das interações: as abelhas (Hymenoptera). No cenário da sinalização e aprendizado, as abelhas são excelentes modelos em estudos de ecologia sensorial e cognitiva, principalmente pela alta capacidade de aprendizagem e variabilidade comportamental, consequência dos diferentes modos de vida (sociais e solitárias) e da diversidade de ambientes que habitam e nos quais forrageiam (Chittka \& Thomson 2001, Loukola et al. 2017). Com o intuito de expandir a percepção da relevância desses processos e padrões nos estudos das interações entre plantas e abelhas visitantes florais, selecionamos os principais componentes de diferentes canais sensoriais conhecidos na literatura, em maior ou menor escala, que influenciam a atividade de forrageio das abelhas. Ressaltamos ainda, o contexto destas interações e sua significância ecológica e evolutiva, com consequências para o sucesso reprodutivo das plantas.

\section{MATERIAL E MÉTODOS}

O material bibliográfico referente aos estudos abordados nas seguintes subseções desta revisão foi obtido através do Portal Periódicos Capes (www.periodicos.capes.gov.br). A busca foi realizada pelo assunto a ser discorrido em cada subseção, utilizando palavras-chave relacionadas com o tema, de forma isolada e/ou combinada, em inglês, tais como: bee, sensory ecology, cognition, anatomy, perception, floral signals, visual system, olfactory system, tactile system, gustatory system, plant chemical signals, petal micromorphology, ectrogmagentic field, e assim por diante. Além dessa busca exata por palavraschave, trabalhos a pirori considerados relevantes sobre os temas de cada subseção também foram incluídos.

\section{PERCEPÇÃO A PARTIR DA VISÃO E A INTERAÇÃO ENTRE CORES, TAMANHOS, FORMAS E SIMETRIA FLORAL}

A atração de visitantes às flores é atribuída em parte à diversidade de cores, formas, tamanhos e simetrias exibidas por suas pétalas e demais componentes florais (Endress 1999). Através destes componentes, as plantas sinalizam a presença de recurso, de maneira honesta ou desonesta, para atrair seus polinizadores (Chittka \& Raine 2006, Pélabon et al. 2012). A variedade de cores, formas, tamanhos e simetrias, bem como seu padrão nas flores, é o resultado de diferentes pressões seletivas, podendo os polinizadores e suas preferências por determinados atributos também atuarem como uma destas (Armbruster $e t$ al. 2005, Herrera et al. 2006, Tanaka \& Brugliera 2006, Koski \& Ashman 2016).

A capacidade visual dos visitantes em detectar e discriminar estímulos florais tem sido foco de muitos estudos nas interações flor-visitante (Chittka \& Menzel 1992, Dyer \& Chittka 2004b, Dyer et al 2011a, Lunau et al. 2011). O sistema visual das abelhas é constituído por três ocelos (conhecidos como olhos simples), usualmente associados com o controle de voo e detecção da intensidade luminosa, e dois olhos compostos formados por centenas de omatídeos (unidade óptica básica) (Land 1977, Snyder 1977, Mizunami 1995, Land \& Nilsson 2002). Cada omatídeo é constituído de uma lente córnea (faceta) que capta a luz direcionando-a para os rabdômeros, estruturas formadas por células fotorreceptoras (Praagh 1980), que por sua vez, determinam a sensibilidade espectral de cada indivíduo/espécie. Nas abelhas, a estrutura interna dos olhos compostos pode se apresentar de duas formas: 1aposição, com capacidade limitada na captura de fótons já que os omatídeos são isolados uns dos outros, de forma que a luz captada atinge apenas o rabdoma correspondente, usualmente encontrado em forrageadores de hábito diurno; e 2superposição, com maior sensibilidade à luz, pois nestes um único rabdoma recebe luz advinda de várias lentes, usualmente encontrado em 
forrageadores de hábitos crepusculares/noturnos (Warrant et al. 2004).

A forma como um visitante percebe visualmente os diferentes sinais florais está relacionada principalmente com a capacidade do seu sistema visual. A maioria das abelhas estudadas até o momento com relação aos seus sistemas visuais, possuem fotorreceptores com sensibilidades máximas em comprimentos de onda na faixa do ultravioleta (UV), azul e verde, o que caracteriza uma visão tricromática (Peitsch et al. 1992, Briscoe \& Chittka 2001), como a dos seres humanos - no entanto, não enxergamos na faixa do UV, mas sim na faixa do vermelho (Nathans 1999). Além do sistema visual, a cor das estruturas florais - ou estruturas de atração em geral como brácteas percebida pelos animais é determinada não apenas pelo comprimento de onda refletido pela superfície das estruturas (reflectância espectral), mas também através da combinação de outros fatores relacionados com $\mathrm{o}$ ambiente onde $\mathrm{o}$ estímulo está presente, como o plano de fundo no qual a estrutura é apresentada (background) e a iluminação ambiente (Kelber et al. 2003, Kemp et al. 2015).

Grande parte das abelhas são ativas em períodos de alta luminosidade durante o dia, mas algumas (cerca de 250 espécies) adquiriram hábitos crepusculares ou noturnos, onde a intensidade de luz é baixa (Kelber et al. 2005, Somanathan et al. 2008a). Embora a maioria dos insetos noturnos apresentem olhos compostos de superposição (mais sensíveis a luz), as abelhas que exibem esse hábito possuem olhos de aposição, comuns às abelhas diurnas. $\mathrm{O}$ que os trabalhos com abelhas noturnas/crepusculares vêm mostrando é que estas exibem adaptações morfológicas em seu sistema visual, apresentando, dentre outras, omatídeos e ocelos maiores em relação àquelas de hábito diurno, o que lhes confere maior sensibilidade e permite forragear em condições de baixa luminosidade (Jander \& Jander 2002, Kelber et al. 2005, Greiner et al. 2006). O significado ecológico dessas adaptações ainda é discutido, mas certamente elas podem conferir vantagens, como a obtenção de maiores quantidades de recursos florais em horários onde a competição seria baixa (Wcislo et al. 2004, Kelber et al. 2005). Para as plantas, a vantagem seria a visita exclusiva de polinizadores, sem a inter- ferência de outros visitantes ( Hopkins et al. 2000, Cordeiro et al. 2016, Soares \& Morellato 2017).

As respostas que permeiam as preferências iniciais de abelhas recém-emergidas (preferências inatas) por determinadas cores podem nos ajudar a entender processos de especializações temporais entre abelhas e plantas. Em condições controladas de laboratório, um estudo com abelhas Apis mellifera L. (1758) (Apidae) recém emergidas mostrou uma preferência destas pelo violeta e azul, cores que por sua vez estavam associadas a flores que apresentavam alta oferta de néctar (Giurfa et al. 1995). No entanto, em outro estudo, a preferência desta mesma espécie e Bombus hortorum L. (1761) forrageando em Tropaeolum majus (Tropaeolaceae), uma espécie polimórfica produzindo flores alaranjadas e amarelas, demonstrou que ambas abelhas exibiram uma preferência pelas flores amarelas em detrimento das alaranjadas, mesmo na ausência de variação no recurso floral (néctar) entre elas (Goulson et al. 2007). Este resultado pode ser explicado pela capacidade de aprendizagem e de discriminação das abelhas. Considerando a cor das flores, o plano de fundo onde elas estavam e o sistema visual das abelhas, flores amarelas são mais facilmente detectadas em comparação com alaranjadas e, se o beneficio é o mesmo, as abelhas devem preferir explorar àquelas onde a relação custo/beneficio é mais vantajosa.

Mudanças na coloração de flores ou estruturas florais são recorrentes na natureza. Existe evidência que esta variação está associada à tarefa de sinalização aos visitantes (Weiss \& Lamont 1997). A mudança de coloração ao longo da antese e retenção de flores senescentes ocorre através de alterações no acúmulo de pigmentos, na manifestação de um pigmento sobre o outro ou até mesmo na combinação destes (Tanaka \& Brugliera 2006, Nishihara \& Nakatsuka 2010, Ohashi et al. 2015). Este mecanismo pode ser desencadeado pela polinização ou programado com a idade da flor (Mathur \& Ram 1978, Ida \& Kudo 2003, Suzuki \& Ohashi 2014). Alguns estudos sugerem que a permanência dessas flores nas plantas incrementam o display floral, contribuindo para a sinalização dos indivíduos (Brito et al 2015, Makino \& Ohashi 2016), além de possivelmente exercerem a função de sinalizadores honestos da presença de recompensa, permitindo aos visi- 
tantes identificarem flores novas, aumentando a eficiência durante as visitas (Ollerton et al. 2007). Como consequência da sinalização visual com o aumento do display, as plantas receberiam mais visitas (Ohashi et al. 2015) e poderiam reduzir a geitonogamia (isto é, transferência de pólen entre flores de um mesmo indivíduo) ao minimizar visitas de abelhas a flores não-receptivas em sinalizações honestas (Schlindwein et al. 2014). No entanto, um estudo com Desmodium setigerum (Fabaceae) mostrou que esta espécie tem a habilidade de reverter seus sinais de mudança de coloração e até mesmo a forma de suas flores quando sofrem limitação polínica (Stanley et al. 2016). Este mecanismo possibilita que as flores que não foram adequadamente polinizadas tenham uma nova chance nesse processo, atraindo outros polinizadores (Willmer et al. 2009).

Além de variações na coloração, a corola pode apresentar diferentes formas de acordo com o número de pétalas, a fusão (ou não) entre elas, o plano de simetria e o formato de suas bordas (Vidal \& Vidal 2006). Assim, o visitante também é capaz de preferir uma flor por sua forma, associada a recompensa oferecida (Blarer et al. 2002, Armbruster et al. 2005, Fenster et al. 2006). Um exemplo é ilustrado no estudo com os principais polinizadores de Erysimum mediohispanicum (Brassicaceae), no qual os principais polinizadores (moscas Bombyliidae, abelhas grandes e pequenas) discriminavam entre as diferentes formas de corola em testes usando flores artificiais sem recompensa. Além disso, as flores com pétalas alongadas (as preferidas pelas abelhas) produziam mais pólen e néctar do que aquelas com pétalas arredondadas, e finalmente, que diferentes populações de plantas eram predominantemente visitadas por uma fauna diferente (Gómez et al. 2008).

Outra importante característica floral envolvida no processo de polinização e discriminação das flores pelos visitantes é a simetria floral (Giurga \& Lehrer 2001). A simetria floral pode ser usada para explicar como determinadas características morfológicas florais se relacionam com a função, no que diz respeito à polinização das plantas (Sprengel 1793). Sprengel (1793) descreveu dois padrões simétricos básicos baseados na organização das estruturas florais, a simetria regular (radial) e irregular (bilateral), também denominadas, respectivamente, de actinomorfa e zigomorfa. A simetria bilateral proporciona maior informação visual, através da complexidade da imagem percebida pelos visitantes em comparação com a simetria radial, intensificando o sinal floral e promovendo o fenômeno de constância nas visitas (veja mais em Davenort \& Kor-ranzadeht 1981). Porém, Leppik (1953) demonstrou uma preferência de Apis mellifera por flores com simetria actinomorfa (radial), enquanto abelhas do gênero Bombus (Apidae, Bombini) apresentaram preferência por formas zigomorfas (bilateral). Posteriormente, foi demonstrado que indivíduos de Apis mellifera treinados com diferentes padrões simétricos apresentaram a capacidade de discriminar e selecionar flores de determinadas simetrias, evidenciando que a simetria floral representa um atributo importante na comunicação planta-abelha (Giurfa et al. 1996). No entanto, a preferência da simetria floral em abelhas ainda não está totalmente esclarecida, principalmente, porque grande parte dos trabalhos que não consideraram as respostas inatas, assim como os diferentes modos de vida (sociais e solitárias) (Neal et al. 1998).

O processo de aquisição da informação visual também é afetado por características intrínsecas das espécies vegetais. O tempo que o visitante floral leva para reconhecer o estímulo depende além da forma, da cor, da iluminação e do plano de fundo, de fatores como tamanho das flores e inflorescências (Spaethe et al. 2001, Skorupski et al. 2006, Telles et al. 2017). Entre os sinais emitidos - por um conjunto de flores (em inflorescências ou não) ou através da unidade floral - existe uma relação positiva entre o tamanho do display, a atração de visitantes e o número de flores visitadas por planta. A taxa de visitação pode aumentar, diminuir ou mostrar uma relação constante com o tamanho do display, tornando o indivíduo planta mais visível e atrativo (Andersson 1988, Klinkhamer \& De Jong 1990, Ohashi \& Yahara 2001, 2002, Mitchell et al. 2004).

Como estratégia de forrageamento ótimo, as abelhas podem usar basicamente dois canais para o processamento da informação visual. Ambos canais são amplamente conhecidos e discutidos no contexto da acurácia e eficiência: o canal neuronal cromático, responsável pelo processamento dos sinais provenientes dos diferentes 
tipos de fotorreceptores das abelhas (UV, azul e verde); e um canal acromático, com capacidade de processar a informação visual de maneira mais rápida e, portanto, sem muitos detalhes, baseado apenas nas diferenças de contraste produzidas entre a flor e o plano de fundo onde esta se encontra (Giurfa et al. 1996, Dyer et al. 2008). A prevalência do uso de um canal ou outro parece depender da distância em que a abelha se encontra do estímulo procurado. Ainda assim, mesmo que a imagem captada a uma determinada distância não permita às abelhas resolverem em detalhes a coloração e padrões florais (Giurfa et al. 1996), a forma floral fornece importantes sinais visuais para que elas consigam discriminar flores de outros objetos presentes no ambiente, ou até mesmo de diferentes espécies a diferentes distâncias (Vorobyev et al. 1997, Ibarra \& Giurfa 2003). A determinação dessas distâncias conhecidas como ângulos visuais - é pouco representativa para a grande maioria das espécies de abelhas, não obstante, considera-se um processo comum a todas elas (Dyer \& Griffiths 2011, Ne'eman \& Ne'eman 2017).

\section{PERCEPÇÃO A PARTIR DO OLFATO}

Os sinais olfativos são percebidos pelas abelhas e outros artrópodes através de estruturas chamadas sensilas, dispostas principalmente nas peças bucais, pernas e antenas (Sutcliffe 1994, Steinbrecht 1996, Hansson \& Stensmyr 2011). Estas estruturas estão relacionadas com diferentes modalidades sensoriais além do olfato, tais como gustação, mecanorrecepção e termorrecepção, mas são principalmente conhecidas pelas funções olfativas e gustativas (Steinbrecht 1996, Spaethe $e t$ al. 2007, Onagbola et al. 2008, Wang et al. 2016), sendo as sensilas multiporosas as principais responsáveis pela função olfativa (BlassioliMoraes et al. 2008, Ravaiano et al. 2014). As sensilas medam a interação química entre receptores proteicos específicos e compostos orgânicos voláteis (COVs ou VOCs, do inglês Volatile Organic Compounds), desencadeando o processamento da informação (Steinbrecht 1996, Stengl et al. 1999, Blassioli-Moraes et al. 2008, Sato \& Touhara 2008; Fialho et al. 2014, Bohbot \& Pitts 2015).
Os VOCs são compostos de baixo peso molecular (e.g. terpenóides, carotenóides, benzenóides e derivados de ácidos graxos) liberados por praticamente todos os tecidos vegetais (Dudareva 2004, Dobson 2006, Dudareva \& Pichersky 2006, Dudareva et al. 2006). Em geral, as fragrâncias florais constituem complexas misturas de VOCs emitidas através de glândulas denominadas osmóforos (Vogel 1963), localizadas em diferentes partes da flor, especialmente nas pétalas (Sazima et al. 1993, Cunningham et al. 2006, Dudareva \& Pichersky 2006, Pansarin et al. 2014). Os VOCs possuem diferentes funcionalidades na interação entre plantas e abelhas, especialmente relacionados à atração floral em si, ou sinalizando a presença de recursos como pólen e néctar (Raguso 2004, Farré-Armengol et al. 2013). Sua presença também pode estar associada a outros processos desencadeados através da interação entre plantas e herbívoros, podendo afetar a sinalização, com consequências para as plantas e seus visitantes (Kessler et al. 2011). Os VOCs variam amplamente em sua presença e abundância relativa em suas misturas, com determinados perfis apresentando associações com certos grupos de visitante floral (Dobson 2006, Jürgens et al. 2006), como por exemplo as abelhas da tribo Euglossini (Apidae). Machos destas abelhas exibem preferência por voláteis que compõem a fragrância floral de plantas de pelo menos dez famílias neotropicais (particularmente representativos em Orchidaceae), o que consequentemente permite uma relação bastante estreita entre seleção de odores florais e preferência por polinizadores (Zimmermann et al. 2009, Hetherington-Rauth \& Ramírez 2016, Mitko et al. 2016, Milet-Pinheiro \& Gerlach 2017).

Algumas espécies de orquídeas produzem fragrância como única recompensa para machos de abelhas Euglossini, concomitantemente utilizado como atrativo floral (Dressler 1982, Sazima et al. 1993, Nunes et al. 2017). Para aquelas espécies co-ocorrendo no tempo e no espaço, exibindo fragrâncias semelhantes e competindo por visitantes, como Gongora bufonia e Catasetum cernuum (Orchidaceae), a posição de deposição do polinário define o sucesso da polinização (Nunes et al. 2017). Espécies distintas podem emitir voláteis através de diferentes partes florais, influenciando o comportamento dos polinizado- 
res durante a coleta para obter um posicionamento ideal dos respectivos polinários (Dodson 1962, Nunes et al. 2017). Desse modo, o compartilhamento de polinizadores - mesmo que geralmente reduzido - não seria problema, já que através desse mecanismo a flor tem diferentes locais tocados pelo visitante durante a visita, o que consequentemente pode aumentar tanto $\mathrm{o}$ sucesso reprodutivo da planta como do polinizador (Hills et al. 1972, Schiestl \& Schlüter 2009, Eltz et al. 2011).

A atração do polinizador mediada pelos VOCs é considerada uma condição derivada porque assume-se que a função original das fragrâncias florais provavelmente era defensiva (Raguso 2004). De maneira generalizada, as fragrâncias florais têm a função de comunicar a presença de um recurso aos polinizadores efetivos e evitar ou repelir visitantes pouco efetivos ou indesejados, atuando como filtros (Raguso 2004, Cunningham et al. 2006, Junker \& Blüthgen 2010). Ainda, as plantas apresentam a capacidade de modular a produção de VOCs dependendo da fase reprodutiva na qual se encontram. Um exemplo clássico dessa modularidade acontece na relação entre formigas e plantas de Acacia spp. (Fabaceae). Neste sistema, as formigas conferem defesa biótica contra herbívoros, mas durante a fase reprodutiva e mais especificamente quando ocorre a abertura das flores, estas passam a produzir VOCs que atuam como repelentes contra as formigas, dissuadindo-as e permitindo a visita de polinizadores. Estas mesmas formigas patrulham os botões jovens e retornam às flores após a deiscência, protegendo os óvulos fertilizados e as sementes em desenvolvimento (Willmer \& Stone 1997).

Assim como no caso das cores, a sinalização das fragrâncias pode ser honesta ou não. Quando honesta, sua presença facilita o reconhecimento pelos forrageadores, especialmente nos casos em que sua intensidade é qualitativa ou quantitativamente correlacionada com a recompensa. Por exemplo, abelhas fêmeas de Osmia (Megachilidae) são capazes de detectar diretamente a presença de néctar nas flores de Penstemon caesius (Plantaginaceae) através dos voláteis presentes no néctar (Howell \& Alarcón 2007). Ainda, os compostos voláteis responsáveis pela fragrância do pólen compreendem voláteis que pertencem às mesmas classes químicas encontradas em aromas de flores, porém contrastando com odores de outras partes florais e indicando a presença do recurso (Dobson \& Bergström 2000). A presença de fragrância em pólen, pétalas e néctar são mais pronunciados nas plantas visitadas por insetos em comparação com aquelas visitadas por aves ou polinizadas pelo vento, sugerindo que a emissão de compostos orgânicos voláteis evoluiu em parte sob seleção para atrair insetos polinizadores (Dobson \& Bergström 2000, Flamini et al. 2002, Schiestl 2015). Assim, a presença de voláteis no néctar e pólen, seria forte e honestamente associada com a presença do recurso, com implicações diretas à biologia reprodutiva das plantas, especialmente à quantidade de visitas que a flor receberia em função da sinalização do próprio recurso (Raguso 2004).

A atração de polinizadores por engano também é bastante frequente na sinalização de VOCs e o único beneficiário dessa interação é a planta. Em diversas espécies de orquídeas, principalmente do gênero Ophrys, as flores mimetizam feromônios sexuais das fêmeas aos machos de diferentes Hymenoptera, como as abelhas, que ao tentar copular com as flores acabam por polinizá-las (Schiestl et al. 1999, Schiestl 2003, 2005, Aysasse et al. 2011).Vale ressaltar ainda, que a sinalização através dos VOCs pode ser alterada caso ocorra a presença de organismos como bactérias e fungos, que podem utilizar os compostos orgânicos presentes no néctar como recurso, afetando a qualidade do néctar através da fermentação e causando repulsa dos polinizadores (Rering et al. 2017).

A emissão de VOCs também pode ocorrer por outras partes das plantas, como sépalas, folhas e frutos (Lucas-Barbosa et al. 2013, Schiestl et al. 2014). Neste caso, a liberação dos VOCs pode acontecer ou ser modificada quanti- e qualitativamente em função da herbivoria, onde os danos nos tecidos causam a liberação de compostos secundários altamente voláteis (Kessler et al. 2011, Pareja et al. 2012, Lucas-Barbosa et al. 2013). Assim, a presença destes compostos, inclusive os liberados nas estruturas florais, seria resultado das pressões seletivas exercidas tanto por polinizadores como por agentes antagonistas (herbívoros e predadores) (Raguso et al. 2003, Zhang et al. 2016), com pequenas alterações modificando a 
preferência dos visitantes, e, consequentemente, o resultado das interações mutualistas (LucasBarbosa et al. 2011, Parachnowitsch et al. 2012). Por exemplo, a herbivoria causa aumento na quantidade de jasmonatos (e.g., Metil Jasmonato) e consequente redução na atratividade floral e aptidão reprodutiva das plantas (Kessler et al. 2011).

Devido à volatilidade dos compostos, existe a possibilidade de sinalização para os visitantes florais a distâncias relativamente maiores que as alcançadas através dos estímulos visuais (Shivanna 2014). Embora alguns trabalhos discordem disso, afirmando que a volatilidade permite comunicação em distâncias mais curtas que os sinais visuais em alguns casos (Dudareva \& Pichersky 2006). Além disso, a presença de fragrâncias permite ainda direcionar polinizadores em ambientes com vegetação densa e com pouca luminosidade, como interior de florestas (Sazima et al. 1993, Paulino-Neto 2014). Uma vantagem da presença de fragrâncias nesses ambientes mais complexos pode ser esperada para visitantes crepusculares ou noturnos, representando bons sinalizadores a esse tipo de visitantes (Warrant 2008, Cordeiro et al. 2017). Em alguns casos as plantas acabam exibindo (e.g., gênero Nicotiana L.) um conjunto de VOCs mais frequentes e intensos durante a noite ou ainda em períodos que permitem maior sucesso reprodutivo, por exemplo, durante as diferentes fases sexuais da flor (Raguso et al. 2003).

\section{PERCEPÇÃO A PARTIR DO TATO}

As antenas das abelhas atuam como órgãos multimodais na captação, através de movimentos ativos (mecanorrecepção), de diferentes informações sensoriais, entre elas, o tato. As sensilas na ponta das antenas estão envolvidas no processo de reconhecimento da espessura e consistência dos sinais relacionados ao recurso (Martin \& Lindauer 1966, Erber et al. 1993). Por exemplo, o padrão de microtextura das pétalas pode variar e essa variação fornece informação relevante para as abelhas, direcionando-as para o centro da flor, onde a recompensa está localizada (Kevan \& Lane 1985).

Existem evidências de que as pernas das abelhas são suficientemente sensíveis para discriminar entre padrões epidérmicos de diferentes espécies vegetais, contribuindo para o seu forrageamento (Kevan \& Lane 1985). Por exemplo, flores com epidermes cônicas são preferidas por abelhas do gênero Bombus, por serem mais rugosas, proporcionando uma melhor aderência do polinizador durante as visitas (Whitney et al. 2008). Esse padrão das pétalas se repete em diversas flores polinizadas por abelhas, diferindo, por exemplo, de flores polinizadas por beija-flores, que tem epidermes com células lisas, associadas talvez ao fato dos beija-flores adejaram em frente as flores e não apresentarem contato direto com as pétalas (Whitney et al. 2008). Além disso, há uma interação entre o estímulo tátil e visual: plantas com diferentes epidermes irão refletir a luz de maneira distinta, produzindo diferenças na intensidade da emissão, ou seja, no brilho percebido pelos visitantes (Whitney et al. 2009).

Assim como nos estudos envolvendo a capacidade de aprendizagem para analisar questões sensoriais visuais e olfativas, a percepção e o aprendizado de pistas táteis também foram investigados em condições controladas, porém não na mesma intensidade (Kevan \& Lane 1985, Menzel et al. 1994, Erber et al. 1998, Scheiner \& Amdam 2009). O que estes estudos demonstraram é que as abelhas são capazes de aprender a associar texturas com recompensas, podendo fazê-lo na natureza. Por exemplo, Erber e colaboradores (1993) verificaram a capacidade de aprendizagem tátil condicionando abelhas Apis mellifera a um determinado padrão de superfície a recompensa encontrada nele. Abelhas podem ser condicionadas a discriminar entre diferentes estruturas superficiais, formas e tamanhos através de movimentos de exploração antenais característicos de reconhecimento (Erber et al. 1993, Scheiner et al. 1999, 2001 a, 2001b, 2003, 2005).

Além da capacidade geral de percepção e resposta aos estímulos, o papel social que desempenham nas colônias pode afetar a aprendizagem tátil em abelhas (Scheiner \& Amdam 2009). O estudo de Scheiner e Amdam (2009) foi pioneiro ao demonstrar que a idade das abelhas apresenta efeitos mínimos na aprendizagem, retenção e discriminação de estímulos táteis, enquanto que o papel social das abelhas dentro da 
colmeia exerce um impacto relevante nas mesmas capacidades. Os autores compararam abelhas operárias forrageadoras e abelhas nutridoras (que permaneceram dentro da colmeia). A aquisição táctil foi comprometida nas abelhas forrageadoras, mas não nas abelhas nutridoras da mesma idade. A função do indivíduo na colmeia (atividade reduzida na colmeia ou atividade de forrageamento ativa fora da colmeia) parece ser o fator que determina a rapidez com a qual uma abelha apresenta sinais de senescência.

\section{PERCEPÇÃO A PARTIR DA GUSTAÇÃO}

A gustação é um importante sentido de percepção dos visitantes florais. É através dessa modalidade sensorial que os visitantes julgam a qualidade dos recursos, decidindo então se a relação será estabelecida. Nas abelhas, as antenas, partes bucais e segmentos distais das pernas dianteiras constituem os principais órgãos quimiossensoriais. Nestes apêndices, células receptoras gustativas, mas também mecanossensoriais e olfativas, estão localizadas dentro de estruturas cuticulares especializadas e mencionadas anteriormente, as sensilas (Brito-Sanchez 2011). A descrição geral das diferentes sensilas gustativas em cada apêndice do corpo das abelhas pode ser encontrada em uma compilação feita por BritoSanchez (2011).

A capacidade de resposta a diferentes concentrações e compostos dos recursos florais não é fixa, variando entre colmeias e indivíduos, assim como no tempo e no espaço. Isso porque as composições nutricionais de compostos orgânicos como proteínas, lipídios e carboidratos, variam de acordo com a espécie de planta (Cnaani et al. 2005, Hanley et al. 2008, Agostini et al. 2014). Por exemplo, o pólen de plantas que dependem da polinização por abelhas possui maior porcentagem de proteínas do que plantas com autopolinização espontânea (Hanley et al. 2008). Como esperado, a composição desses recursos florais afeta diretamente a frequência de visitantes de acordo com experiências prévias, já que as abelhas podem perceber e selecionar o recurso (Cnaani et al. 2005). Por outro lado, pode ocorrer uma fidelização de visitas, por parte das abelhas, em flores com morfologia semelhante àquela em que houve experiência prévia positiva quanto à qualidade do recurso (Muth et al. 2018). Além disso, a complexidade da vida social das abelhas, como nas colônias de Apis mellifera, reforça o papel da gustação, atuando no reconhecimento interindividual e na variedade de compostos aos quais as abelhas são expostas durante as diferentes atividades realizadas. Alguns estudos recentes relatam a incapacidade de abelhas em reconhecer compostos neonicotinoides, consequentemente, afetando a seleção dos recursos (contaminados e não contaminados) durante o forrageamento (Kessler et al. 2015, Raine \& Gill 2015).

Um dos principais desafios da sinalizaçao floral é que os sinais devem ser não apenas atraentes, mas também memoráveis: quanto mais distinto um sinal for, mais provável é que um polinizador se lembre dele, aumentando a chance de polinizadores visitarem mais flores de determinadas espécies enquanto ignoram outras concorrentes (Chittka \& Peng 2013). Wright e colaboradores (2013) relatam que algumas espécies de plantas parecem se beneficiar neste ambiente competitivo, manipulando a memória das abelhas com substâncias neuroestimulantes, como a cafeína e a nicotina, presentes no néctar (Raguso 2008, Wright et al. 2013). Estes estimulantes, produzidos como compostos secundários, são substâncias de baixo investi-mento para as plantas, mas com efeitos intensos para os visitantes (Chittka \& Peng 2013). Por exemplo, espécies contendo cafeína recebem mais visitantes independente da coloração floral (Thomson et al. 2015), além de gerarem dependência, compromentendo a entrada de recursos na colmeia (Raguso 2008, Chittka \& Peng 2013, Wright et al. 2013). Originalmente, ocorrem em muitos tecidos vegetais (atuando na defesa contra herbívoros), podendo ser adicionados facilmente ao néctar, além de serem encontrados em espécies pertencentes a diferentes famílias como Solanaceae (Nicotiana L.), Tiliaceae (Tilia cordata Mill), Rutaceae (Citrus L.), Rubiaceae (Coffea L.) e Asteraceae (Helianthus annuus).

Se por um lado os polinizadores são estimulados a incrementar o número de visitas através da 
presença de compostos secundários, os pilhadores de Nicotiana attenuata (Solanaceae) realizam menos visitas (Kessler et al. 2008), assim como em Catalpa speciosa (Bignoniaceae), devido a presença de substâncias tóxicas no néctar (Stephenson 1981). Determinados compostos secundários presentes nos recursos florais podem ser tóxicos para visitantes não especializados na coleta desse recurso (Adler 2000, Kessler et al. 2008). Tais compostos também são capazes de reduzir a ação de microrganismos que causam contaminação do néctar e afetam de alguma forma as plantas e os visitantes florais (Adler 2000, Herrera et al. 2008). Portanto, compostos secundários geralmente tendem a aumentar $\mathrm{o}$ sucesso reprodutivo das plantas ao atrair um maior número de visitas de abelhas (Singaravelan et al. 2005), e otimizar o comportamento de polinizadores (Kessler et al. 2008, Wright et al. 2013), potencializando a memória do estimulo e seu recurso, gerando, assim, constância (Chittka \& Peng 2013, Wright et al. 2013). No entanto, a presença de compostos secundários e seus efeitos diretos em diferentes grupos de visitantes florais ainda é um tema que se restringe a poucos estudos na literatura.

A resposta das abelhas a quantidade do recurso pode se tornar irrelevante frente a qualidade do mesmo. A frequência de visitas em flores com diferentes volumes ofertados de néctar pode ser a mesma, mas o contrário não se aplica, o que demonstra que a percepção da concentração é mais relevante do que a percepção do volume para esses visitantes (Cnaani et al. 2005). Além disso, o néctar apresenta não apenas diferentes tipos de açúcares, como sacarose, glicose e/ou frutose, mas também ácidos orgânicos, lipídios, minerais, vitaminas e compostos aromáticos (Brito-Sanchez et al. 2007). Já foi demonstrado que abelhas com probóscide longa, comumente, preferem néctar rico em sacarose, enquanto que abelhas com probóscide curta e moscas preferem néctar rico em hexoses (Nepi et al. 2009). Por outro lado, abelhas rejeitam soluções com forte amargor e com alta salinidade (Sanchez 2011), uma descoberta que influencia a execução de muitos experimentos comportamentais, nos quais o erro na escolha de um estímulo leva a penalidade de provar substâncias amargosas pelas abelhas (Rodríguez-Gironés et al. 2013).

\section{PERCEPÇÃO A PARTIR DO ELETROMAGNETISMO}

No contexto histórico da biologia da polinização, o eletromagnetismo se apresenta como uma modalidade sensorial recém-descoberta utilizada no processo de comunicação entre plantas e seus visitantes florais. Abelhas possuem um senso triboelétrico de percepção mediado por mecanorreceptores presentes nas antenas e localizados nos pelos filiformes (Clarke et al. 2013, Greggers et al. 2013, Sutton et al. 2016, Zakon 2016, Clarke et al. 2017). O mecanismo respeita leis naturais de cargas positivas e negativas com respeito aos objetos estáticos e em movimento. Como resultado da sua interação com o meio, os insetos carregam diferentes cargas elétricas que podem influenciá-los de diversas formas (Edwards 1962).

No caso das abelhas, a influência pode ser expressa no comportamento de termorregulação da colônia, na comunicação e navegação dos indivíduos (Warnke 1976). No estudo pioneiro de Warnke (1976), as colônias de Apis mellifera foram submetidas a diferentes frequências de campos elétricos e verificaram que as abelhas submetidas a campos elétricos fortes se tornaram agressivas e apresentaram problemas de comunicação, além disso aumentaram a temperatura da colônia anormalmente. No que diz respeito à comunicação, ao realizarem contato antenal uma corrente elétrica flui de uma abelha para a outra. Dependendo da resistência interna no corpo da abelha, esta corrente terá diferente força. O autor especula que dessa forma as abelhas seriam capazes de reconhecer abelhas de sua colônia, visto que as de outras colônias apresentariam resistências diferentes (Warnke 1976).

Um determinado objeto se torna eletrostáticamente carregado quando tem elétrons adicionados a ele (se tornando negativamente carregado) ou quando tem elétrons removidos (tornando-se positivamente carregado), além disso cargas elétricas opostas se atraem (Vaknin $e t$ al. 2000). De maneira geral, as flores apresentam naturalmente cargas elétricas negativas, devido à influência das cargas positivas do ar que se acumulam em seu entorno, induzindo tais cargas para às pétalas (Cobert et al. 1982, Bowker \& Crenshaw 2007, Clarke et al. 2013, Clarke et al. 2017). Já as abelhas apresentam carga positiva 
(Erickson 1975, Warnke 1976), por isso, quando a abelha pousa na flor, parte da carga positiva em seu corpo cancela parte das cargas negativas presentes na flor, alterando a conformação do campo elétrico, fenômeno perceptível para a próxima abelha que busca visitar a flor (Clarke $e t$ al. 2013). A distribuição do campo elétrico na planta varia com o formato das pétalas, sendo complexa, não uniforme e mudando rapidamente devido a corrente condutora que flui da terra (Cobert et al. 1982, Dai \& Law 1995).

Alguns estudos evidenciam a capacidade das abelhas do gênero Bombus e Apis mellifera em perceber e se comunicar por meio de campos elétricos (Warnke 1976, Kirschvink \& Gould 1981, Colin et al. 1991, Clarke et al. 2013, Greggers et al. 2013). Além disso, não somente se tem mostrado a capacidade das abelhas em reconhecer campos eletromagnéticos presentes nas flores (Clarke et al. 2013, Greggers et al. 2013, Sutton et al. 2016), mas como tal percepção pode ser uma ferramenta de comunicação rápida e dinâmica entre planta e polinizador (Clarke et al. 2013, Brito et al. 2014). Uma das hipóteses levantadas para explicar a relevância dos sinais eletromagnéticos é o uso desta informação sensorial na identificação de flores que foram recentemente visitandas. A percepção da mudança de carga dos campos elétricos aumentaria a eficácia das abelhas no momento de selecionar em quais flores pousar e coletar recurso (Clarke et al. 2013, Zakon 2016, Clarke et al. 2017). A espécie Bombus terrestris, por exemplo, pode detectar e aprender como utilizar a variação estrutural do campo elétrico floral para discriminar entre flores visitadas ou não visitadas (Clarke et al. 2013). Ao que tudo indica, a percepção do campo elétrico faz parte da experiência de forrageamento dessa espécie, visto que a velocidade de aprendizado dessas abelhas aumenta quando esse sentido está pareado com outros, como a cor (Clarke et al. 2013, 2017).

\section{CONCLUSÃO}

Os componentes fundamentais que medeiam as interações entre flores e seus visitantes (seja através de relações generalistas, especialistas, mutualistas ou antagonistas) podem ser divididos em dois elementos principais: as capacidades sensoriais e cognitivas dos visitantes e os sinais florais emitidos pelas diferentes espécies vegetais, associados, posteriormente, com o valor dos recursos oferecidos (Riffell 2011). Plantas disputam a atenção de seus polinizadores através de uma diversidade de atributos. A exibição da flor e seus recursos, envolvendo odor, cor, morfologia, textura e sabor, são todos direcionados para estimular o sistema sensorial dos possíveis visitantes, especialmente aqueles com potencial para promover a polinização.

O que parece ser mais comum no processo da sinalização é que exista um contínuo na maneira como os animais podem perceber os estímulos. Às vezes, a presença de um único sinal em uma única modalidade pode ser suficientemente potente para desencadear respostas satisfatórias nas abelhas (Telles et al. 2017). Com exceção de alguns exemplos (Leonard \& Papaj 2011, Sánchez et al. 2011, Katzenberger et al. 2013, Leonard et al. 2013, Leonard \& Masek 2014), estudos detalhando a maneira como as abelhas respondem às diferentes sinalizações, simples ou complexas, são poucos, e os caminhos evolutivos que levaram a um tipo ou outro de sinalização ainda não são tão claros, podendo ser contexto-dependentes. Devemos lembrar que os sinais também enfrentam seleção, não só do ambiente físico (Koski \& Ashman 2015), mas também dos sistemas sensoriais e cognitivos dos receptores, através das preferências (inatas e/ou aprendidas) e do trade-off entre gastos energéticos e a energia obtida. $\mathrm{O}$ produto final dessa relação entre sinalizações simples e complexas e a motivação dos visitantes pode ser medido através do sucesso reprodutivo de ambos interagentes (Kulahci et al. 2008), onde plantas teriam sua reprodução favorecida pela transferência de grãos de pólen - no caso de agentes atuando como polinizadores - para um estigma coespecífico (Waser 1978, Chittka et al. 2001), enquanto visitantes receberiam em troca nos processos honestos de sinalização - o recurso pelo qual buscam.

Atributos comuns a uma infinidade de espécies vegetais, como a simetria e a morfologia floral, podem desempenhar um papel de extrema relevância em diferentes processos como, por exemplo, de isolamento reprodutivo e constância por parte dos visitantes. Ao que parece, a maioria das espécies com uma simetria irregular foram 
derivadas de espécies com flores com simetria regular (Neal et al. 1998). Em que circunstâncias a presença de flores com simetrias irregulares representa uma vantagem para ambos interagentes? Ou até que ponto diferentes morfologias florais são o resultado de pressões bióticas e não abióticas? A evolução de uma corola bilateralmente simétrica poderia restringir a direção de aproximação e movimento de polinizadores nas flores, fazendo com que a visita e coleta do recurso fosse apenas possível a partir de uma única direção, o que facilita a deposição específica de pólen no corpo do polinizador e o contato mais preciso com o estigma, por exemplo (Schlindwein et al. 2014). Quando combinada com o tamanho, morfologia e constância do polinizador, a deposição em áreas especificas pode aumentar a probabilidade de que grãos de pólen alcancem um estigma compatível (Sargent 2004). Além disso, as flores podem ter diferentes padrões geométricos de disposição do campo elétrico, determinados pela sua morfologia, que são distinguíveis pelas abelhas, podendo atuar como sinalizadores (Clarke et al. 2013).

A restrição de células cônicas às pétalas das flores leva à sugestão de que elas estão envolvidas na atração de polinizadores (Whitney et al. 2009). No entanto, a existência de diferenças e o papel da microtextura de pétalas ainda é pouco explorada nos estudos das interações. Sinais táteis de microtextura nas pétalas podem atuar como guias de néctar para as abelhas, por exemplo, com ou sem a diferenciação visual, uma função que poderia ser testada em plantas visitadas por animais que forrageiam durante períodos de baixa luminosidade, como abelhas crepusculares e noturnas, ou ainda para plantas que apresentem orientações especificas de suas flores (upside down).

No contexto de forrageamento, a determinação da qualidade do néctar e pólen, que fornecem, respectivamente, carboidratos e proteínas (entre outros), irá influenciar a sobrevivência dos indivíduos e da prole, contudo, o entendimento das demandas de diferentes grupos de abelhas (sociais e solitárias) está restrito a poucas espécies. No caso dos VOCs, alguns compostos podem ser muito específicos e raros, enquanto outros especialmente comuns. Por exemplo, o composto aromático benzaldeído e o monoterpeno linalol foram encontrados em mais de $50 \%$ das famílias de plantas investigadas até o momento (Knudsen et al. 2006). Da mesma forma como acontece para a maioria dos sinais, no entendimento da relação entre VOCs e a resposta das abelhas ainda existem muitas perguntas em aberto.

Muito do que sabemos sobre sistemas sensoriais e cognitivos em abelhas se restrige a duas espécies: Apis mellifera e Bombus terrestris. Se considerarmos a diversidade de habitats, estilos de vida, tempos de divergência, hábitos de forrageamento e as cerca de 16.000 espécies restantes, podemos concluir que nosso entendimento é limitado (Michener 2000). Como mensagem final, gostaríamos de incentivar os leitores a explorarem essa diversidade e que incorporassem as questões sensoriais e cognitivas em seus estudos de interações, para uma melhor compreensão da diversidade de comportamentos e respostas aos diferentes sinais florais, variando no tempo e no espaço.

\section{AGRADECIMENTOS}

Este trabalho é resultado da disciplina "Fundamentos e Fronteiras da Ecologia da Polinização", oferecida pelos programas de Pós-Graduação em Biologia Vegetal e Ecologia e Conservação de Recursos Naturais da Universidade Federal de Uberlândia. Todos os autores são gratos pela oportunidade de aprendizado durante a escrita e revisão deste trabalho. Agradecemos aos três revisores anônimos que contribuíram para melhoria desse manuscrito. LRFM, BMCG, LCO e TNA agradecem à CAPES pelas respectivas bolsas de mestrado. GJB agradece pela bolsa PELD/ CAPES/CNPq $\quad n^{\circ} \quad 88887.137914 / 2017-00 . \quad$ FJT agradece pela bolsa PNPD/Capes.

\section{REFERÊNCIAS}

Adler, L. S. 2000. The ecological significance of toxic nectar. Oikos, 91(3), 409-420. DOI: 10.1034/j.1600-0706.2000.910301.x

Agostini, K., Sazima, M., \& Galetto, L. 2011. Nectar production dynamics and sugar composition in two Mucuna species (Leguminosae, Faboideae) with different specialized pollinators. Naturwis- 
senschaften, 98(11), 933-942. DOI: $10.1007 / \mathrm{s} 00$ 114-011-0844-6

Amaya-Márquez, M. 2009. Floral constancy in bees: a revision of theories and a comparison with other pollinators. Revista Colombiana de Entomologia, 35(2), 206-216.

Andersson, S. 1988. Size-dependent pollination efficiency in Anchusa officinalis (Boraginaceae): causes and consequences. Oecologia, 76, 125130.

Araújo, A. C., \& Sazima, M. 2003. The assemblage of flowers visited by hummingbirds in the “capões” of Southern Pantanal, Mato Grosso do Sul, Brazil. Flora-Morphology, Distribution, Functional Ecology of Plants, 198(6), 427-435.

Armbruster, W. S., Antonsen, L., \& Pelabon, C. 2005. Phenotypic selection on Delachampia blossoms: honest signaling affects pollination success. Ecology, 86, 3323-3333. DOI: 10.189 0/04-1873

Assunção, M. A., Torezan-Silingardi, H. M., \& DelClaro, K. 2014. Do ant visitors to extrafloral nectaries of plants repel pollinators and cause an indirect cost of mutualism? Flora: Morphology, Distribution, Functional Ecology of Plants, 209(5-6), 244-249. DOI: 10.1016/j. flora.2014.03.003

Ayasse, M., Stökl, J., \& Francke, W. 2011. Chemical ecology and pollinator-driven speciation in sexually deceptive orchids. Phytochemistry, 72(13), 1667-1677. DOI: 10.1016/j.phytochem. 2011.03.023

Ballantyne, G., \& Willmer, P. 2012. Nectar theft and floral ant-repellence: a link between nectar volume and ant-repellent traits? PLoS ONE, 7(8), e43869. DOI: 10.1371/journal.pone.00438 69

Barônio, G. J., Maciel, A. A., Oliveira, A. C., Kobal, R. O. A. C., Meireles, D. A. L., Brito, V. L. G., \& Rech, A. R. 2016. Plantas, polinizadores e algumas articulações da biologia da polinização com a teoria ecológica. Rodriguésia, 67(2), 275293. DOI: 10.1590/2175-7860201667201

Bergamo, P. J., Wolowski, M., Maruyama, P. K., Vizentin-Bugoni, J., Carvalheiro, L. G., \& Sazima, M. 2017. The potential indirect effects among plants via shared hummingbird pollinators are structured by phenotypic similarity. Ecology. In press. DOI: 10.1002/ecy. 1859
Blande, J. D., \& Glinwood, R. 2016. Deciphering chemical language of plant communication. Springer International Publishing, Cham: p. 325. DOI: 10.1007/978-3-319-33498-1

Blarer, A., Keasar, T., \& Shmida, A. 2002. Possible mechanisms for the formation of flower size preferences by foraging bumblebees. Ethology, 108, 341-351. DOI: 10.1046/j.1439-0310.2002.0 0778.x

Blassioli-Moraes, M. C., Laumann, R. A., Paula, D. P., Pareja, M., Silva, C. C. A., Viera, H. G., Naime, J. M., \& Borges, M. 2008. Eletroantenografia - a antena do inseto como um biossensor. no. 270 . Brasília: Documentos Embrapa - Recursos Genéticos e Biotecnologia: p. 22.

Bohbot, J. D., \& Pitts, R. J. 2015. The narrowing olfactory landscape of insect odorant receptors. Frontiers in Ecology and Evolution, 3, 39. DOI: 10.3389/fevo.2015.00039

Bowker, G. E., \& Crenshaw, H. C. 2007. Electrostatic forces in wind-pollination-Part 1: Measurement of the electrostatic charge on pollen. Atmospheric Environment, 41(8), 15871595. DOI: 10.1016/j.atmosenv.2006.10.047

Briscoe, A. D., \& Chittka, L. 2001. The evolution of color vision in insects. Annual Review of Entomology, 46(1), 471-510.

Brito, V. L. G., Telles, F., \& Lunau, K. 2014. Ecologia cognitiva da polinização. In: A. R. Rech, K. Agostini, P. E. A. M. Oliveira, \& I. C. Machado (Eds.), Biologia da Polinização. Rio de Janeiro: Editora Projeto Cultural.

Brito, V. L., Weynans, K., Sazima, M., \& Lunau, K. 2015. Trees as huge flowers and flowers as oversized floral guides: the role of floral color change and retention of old flowers in Tibouchina pulchra. Frontiers in Plant Science, 6, 362. DOI: 10.3389/fpls.2015.00362

Brito, V. L. G., Pinheiro, M., \& Sazima, M. 2010. Sophora tomentosa e Crotalaria vitellina (Fabaceae): Biologia reprodutiva e interações com abelhas na restinga de Ubatuba, São Paulo. Biota Neotropica, 10(1), 185-192.

Brito-Sanchez, M. G., Giurfa, M., Paula Mota, T. R., \& Gauthier, M. 2005. Electrophysiological and behavioural characterization of gustatory responses to antennal "bitter" taste in honeybees. European Journal of Neuroscience, 22, 31613170. DOI:10.1111/j.1460-9568.2005.04516.x

Brito-Sanchez, M. G., Serre, M., Avarguès-Weber, 
A., \& Dyer, A. G. 2015. Giurfa M. Learning context modulates aversive taste strength in honey bees. Journal of Experimental Biology Mar, 218(6), 949-59. DOI: 10.1242/jeb.117333.

Brito-Sanchez, M. G. 2011. Taste perception in honey bees. Chemical Senses, 36(8), 675-692. DOI: 10.1093/chemse/bjr040

Brito-Sanchez, M. G., Ortigão-Farias, J. R., Gauthier, M., Liu, F. L. \& Giurfa, M. 2007. Taste perception in honeybees: just a taste of honey? Arthropod Plant Interact, 1, 69-76. DOI: 10. 1007/s11829-007-9012-5

Brodie, B. S., Smith, M. A., Lawrence, J., \& Gries, G. 2015. Effects of floral scent, color and pollen on foraging decisions and oocyte development of common green bottle flies. PLOS ONE, 10(12), e0145055. DOI: 10.1371/journal.pone.0145055

Bro-Jørgensen, J. 2010. Dynamics of multiple signalling systems: animal communication in a world in flux. Trends in Ecology and Evolution, 25(5), 292-300. DOI: 10.1016/j.tree.2009.11.003

Bronstein, J. L., Barker, J. L., Lichtenberg, E. M., Richardson, L. L., \& Irwin, R. E. 2017. The behavioral ecology of nectar robbing: why be tactic constant? Current Opinion in Insect Science, 21, 14-18. DOI: 10.1016/j.cois.2017.05. 013

Burger, H., Dötterl, S., \& Ayasse, M. 2010. Hostplant finding and recognition by visual and olfactory floral cues in an oligolectic bee. Functional Ecology, 24, 1234-1240. DOI: 10.11 11/j.1365-2435.2010.01744.x

Burgett, M., Sukumalanand, P., \& Vorwohl, G. 2005. Pollen species resources for Xylocopa (Nyctomelitta) tranquebarica (F.), a night-flying carpenter bee (Hymenoptera: Apidae) of Southeast Asia. Science Asia, 31, 65-68.

Burkle, L. A., \& Runyon, J. B. 2017. The smell of environmental change: Using floral scens to explain shifts in pollinator attraction. Applications in Plant Sciences, 5(6), 1600123. DOI: 10.3732/apps.1600123

Campan, R., \& Lehrer, M. 2002. Discrimination of closed shapes by two species of bee, Apis mellifera and Megachile rotundata. Journal of Experimental Biology, 205(4), 559-572.

Cembrowski, A. R., Tan, M. G., Thomson, J. D., \& Frederickson, M. E. 2014. Ants and ant scent reduce bumblebee pollination of artificial flowers. The American Naturalist, 183(1), 133-
139. DOI: $10.1086 / 674101$

Chittka, L., \& Menzel, R. 1992. The evolutionary adaptation of flower colours and the insect pollinators' colour vision. Journal of Comparative Physiology A: Neuroethology, Sensory, Neural, and Behavioral Physiology, 171(2), 171-181.

Chittka, L., \& Peng, F. 2013. Caffeine boosts bees' memories. Science, 339(6124), 1157-1159. DOI: $10.1126 /$ science.1234411

Chittka, L., \& Raine, N. E. 2006. Recognition of flowers by pollinators. Current Opinion in Plant Biology, 9(4), 428-435.

Chittka, L., Ings, T. C., \& Raine, N. E. 2004. Chance and adaptation in the evolution of island bumblebee behaviour. Population Ecology, 46, 243-251. DOI: 10.1007/s10144-004-0180-1

Chittka, L., Thomson, J. D., \& Waser, N. M. 1999. Flower constancy, insect psychology, and plant evolution. Naturwissenschaften, 86(8), 361-377. DOI: $10.1007 / \mathrm{s} 001140050636$

Chittka, L., \& Thomson, J. D. 2001. Cognitive ecology of pollination. Cambridge: Cambridge Univeristy Press: p. 344.

Clarke, D., Morley, E., \& Robert, D. 2017. The bee, the flower, and the electric field: electric ecology and aerial electroreception. Journal of Comparative Physiology A, 203(9), 737-748. DOI 10. 1007/s00359-017-1176-6

Clarke, D., Whitney, H., Sutton, G., \& Robert, D. 2013. Detection and learning of floral electric Fields by Bumblebees. Science, 340, 66-70. DOI: $10.1126 /$ science. 1230883

Cnaani, J., Thomson, J. D., \& Papaj, D. R. 2006. Flower choice and learning in foraging bumblebees: effects of variation in nectar volume and concentration. Ethology, 112(3), 278-285, DOI: 10.1111/j.1439-0310.2006.01174.x

Cobert, S. A., Beament, J., \& Eisikowitch, D. 1982. Are electrostatic forces involved in pollen transfer? Plant, Cell \& Environment, 5(2), 125129. DOI: $10.1111 / 1365-3040$.ep1 1571488

Colin, M. E., Richard, D., \& Chauzy, S. 1991. Measurement of eletric charges carried by bees: evidence of biological variations. Journal of Bioelectricity, 10(2), 17-32.

Collier, K. F., Albuquerque, G. S., Eiras, A. E., Blackmer, J. L., Araujo, M. C., \& Monteiro, L. B. 2001. Estímulos olfativos envolvidos na localização de presas pelo ácaro predador 
Neoseiulus californicus (McGregor) (Acari: Phytoseiidae) em macieiras e plantas hospedeiras alternativas. Neotropical Entomology, 30(4), 631-639.

Cordeiro, G. D., Pinheiro, M., Dötterl, S., \& Alvesdos-Santos, I. 2016. Pollination of Campomanesia phaea (Myrtaceae) by nightactive bees: a new nocturnal pollination system mediated by floral scent. Plant Biology, 19(2), 132-139. DOI: $10.1111 /$ plb. 12520

Cozzolino, S., Fineschi, S., Litto, M., Scopece, G., Trunschke, J., \& Schiestl, F. P. 2015. Herbivory increases fruit set in Silene latifolia: A consequence of induced pollinator-attracting floral volatiles? Journal of Chemical Ecology, 41(7), 622-630. DOI: 10.1007/s10886-015-0597-3

Crepet, W. L., Friis, E. M., \& Nixon, K. C. 1991. Fossil evidence for the evolution of biotic pollination. Philosophical Transactions of the Royal Society of London B Biological Sciences, 333, 187-95.

Cunningham, J. P., Moore, C. J., Zalucki, M. P., \& Cribb, B. W. 2006. Insect odour perception: recognition of odour components by flower foraging moths. Proceedings of the Royal Society B: Biological Sciences, 273(1597), 20352040. DOI: $10.1098 / \mathrm{rspb} .2006 .3559$

Dai, Y., \& Law, S. E. 1995. Modeling the transient electric field produced by a charged pollen cloud entering a flower. IEEE/IAS Conference, 2, 1395-1402. DOI: 10.1109/IAS.1995.530465

Daly, K., Smith, B., \& Wright, G. 2006. Learningbased recognition and discrimination of floral odors. In: N. Dudareva, \& E. Pichersky, Biology of Floral Scent. pp. 263-295. CRC Press. DOI: 10.1201/9781420004007.ch12

Dangles, O., Irschick, D. J., Chittka, L., \& Casas, J. 2009. Variability in sensory ecology: expanding the bridge between physiology and evolutionary biology. The Quarterly Review of Biology, 84(1), 51-74. DOI: 10.1086/596463

Davenport, D., \& Kohanzadeh, Y. 1982. Orchids, bilateral symmetry and insect perception. Journal of Theoretical Biology, 94(2), 241-252. DOI: 10.1016/0022-5193(82)90311-3

Delle-Vedove, R., Juillet, N., Bessière, J.-M., Grison, C., Barthes, N., Pailler, T., \& Schatz, B. 2011. Colour-scent associations in a tropical orchid: Three colours but two odours. Phytochemistry, 72(8), 735-742. DOI: 10.1016/j.phytochem.2011.
02.005

Dobson, H. E. M., \& Bergström, G. 2000. The ecology and evolution of pollen odors. Plant Systematics and Evolution, 222(1-4), 63-87. DOI: $10.1007 / \mathrm{BF} 00984096$

Dobson, H. E. M. 2006. Relationship between floral fragrance composition and type of pollinator. In: N. Dudareva \& E. Pichersky (Eds.), Biology of Floral Scent. pp. 147-198. Taylor Francis Group, New York.

Dodson, C. H. 1962. The importance of pollination in the evolution of the orchids of tropical America. Bulletin American Orchid Society, 31, 525-534.

Dormont, L., Delle-Vedove, R., Bessière, J.-M., \& Schatz, B. 2014. Floral scent emitted by white and coloured morphs in orchids. Phytochemistry, 100, 51-59. DOI: 10.1016/j.phy tochem.2014.01.009

Dressler, R. L. 1982. Biology of the orchid bees (Euglossini). Annual Review of Ecology and Systematics, 13(1), 373-394.

Dudareva, N. 2004. Biochemistry of Plant Volatiles. Plant Physiology, 135(4), 1893-1902. DOI: 10.1104/pp.104.049981

Dudareva, N., \& Pichersky, E. 2006. Floral scent metabolic pathways: Their regulation and evolution. In: N. Dudareva \& E. Pichersky (Eds.), Biology of Floral Scent. pp. 55-78. Boca Raton, FL: CRC Press.

Dudareva, N., Negre, F., Nagegowda, D. A., \& Orlova, I. 2006. Plant Volatiles: Recent Advances and Future Perspectives. Critical Reviews in Plant Sciences, 25(5), 417-440. DOI: 10.1080/07 352680600899973

Dukas, R. 2004. Evolutionary Biology of Animal Cognition. Annual Review of Ecology, Evolution, and Systematics, 35, 347-374. DOI: 10.1146/ annurev.ecolsys.35.112202.130152

Dukas, R. \& Ratcliffe, J. M. 2009. Cognitive Ecology II. The University of Chicago Press, Chicago. p. 384.

Dyer, A. G., \& Chittka, L. 2004. Biological significance of discriminating between similar colours in spectrally variable illumination: bumblebees as a study case. Journal of Comparative Physiology A, 190, 105-114. DOI: 10.1007/s00359-003-0475-2

Dyer, A. G., \& Chittka, L. 2004b. Bumblebees (Bombus terrestris) sacrifice foraging speed to 
solve difficult colour discrimination tasks. Journal of Comparative Physiology A, 190, 759763.

Dyer, A. G., Spaethe, J. \& Prack, S. 2008. Comparative psuchophysics of bumblebee and honeybee colour discrimination and object detection. Journal of Comparative Physiology A, 194, 617-627.

Dyer, A. G., \& Griffiths, D. W. 2011. Seeing near and seeing far; behavioural evidence for dual mechanisms of pattern vision in the honeybee (Apis mellifera). The Journal of Experimental Biology, 215, 397-404. DOI: 10.1242/jeb.060954

Dyer, A. G., Paulk, A. C., \& Reser, D. H. 2011. Colour processing in complex environments: insights from the visual system of bees. Proceedings of the Royal Society of London B: Biological Sciences, 278(1707), 952-959. DOI: $10.1098 / \mathrm{rspb} .2010 .2412$

Edwards, D. K. 1962. Electrostatic charges on insects due to contact with different substrates. Canadian Journal of Zoology, 40(845), 579-584. DOI: 10.1139/z62-051

Ellis, A. G., \& Johnson, S. D. 2010. Floral mimicry enhances pollen export: the evolution of pollination by sexual deceit outside of the orchidaceae. The American Naturalist, 176(5), E143-E151. DOI: 10.1086/656487

Eltz, T., Fritzsch, F., Pech, J. R., Zimmermann, Y., Ramírez, S. R., Quezada-Euan, J. J. G., \& Bembé, B. 2011. Characterization of the orchid bee Euglossa viridissima (Apidae: Euglossini) and a novel cryptic sibling species, by morphological, chemical, and genetic characters. Zoological Journal of the Linnean Society, 163(4), 10641076. DOI: $10.1111 /$ j.1096-3642.2011.00740.x

Eltz, T., Roubik, D. W., \& Lunau, K. 2005. Experience-dependent choices ensure speciesspecific fragrance accumulation in male orchid bees. Behavioral Ecology and Sociobiology, 59(1), 149-156. DOI: 10.1007/s00265-005-0021-z

Endler, J. A., \& Basolo, A. L. 1998. Sensory ecology, receiver biases and sexual selection. Trends in Ecology and Evolution, 13(10), 415-420. DOI: 10.1016/S0169-5347(98)01471-2

Endress, P. K. 1994. Diversity and evolutionary biology of tropical flowers, Oxford, Pergamon Press. Furness, p. 511.

Endress, P. K. 1999. Patterns of floral construction in ontogeny and phylogeny. Biological Journal of the Linnean Society, 39, 153-175.

Erber, J., Kierzek, S., Sander, E., \& Grandy, K. 1998. Tactile learning in the honeybee. Journal of Comparative Physiology A, 183, 737. DOI: 10.1007 / s003590050296

Erber, J., Pribbenow, B., Bauer, A., \& Kloppenburg, P. 1993. Antennal refl exes in the honeybee tools for studying the nervous-system. Apidologie, 24(3), 283-296.

Erickson, E. H. 1975. Surface electric potentials on worker honeybees leaving and entering the hive. Journal of Apicultural Research, 14(3-4), 141-147. DOI: 10.1080/00218839.1975.11099818

Evoy, W. H., \& Jones, B. P. 1971. Motor patterns of male euglossine bees evoked by floral fragrances. Animal Behaviour, 19, 583-588.

Farré-Armengol, G., Filella, I., Llusia, J., \& Peñuelas, J. 2013. Floral volatile organic compounds: Between attraction and deterrence of visitors under global change. Perspectives in Plant Ecology, Evolution and Systematics, 15(1), 56-67. DOI: 10.1016/j.ppees.2012.12.002

Fenster, C. B., Cheely, G., Dudash, M. R. \& Reynolds, R. J. 2006. Nectar reward and advertisement in hummingbird pollinated Silene virginica (Caryophyllaceae). American Journal of Botany, 93, 1800-1807. DOI: 10.3732/ ajb.93.12.1800.

Fialho, M. do C. Q., Guss-Matiello, C. P., Zanuncio, J. C., Campos, L. A. O., \& Serrão, J. E. 2014. A comparative study of the antennal sensilla in corbiculate bees. Journal of Apicultural Research, 53(3), 392-403. DOI: 10.3896/IBRA. 1.53.3.07

Finger, E., \& Burkhardt, D. 1994. Biological aspects of bird colouration and avian colour vision including ultraviolet range. Vision research, 34(11), 1509-1514.

Flamini, G., Cioni, P. L., \& Morelli, I. 2002. Differences in the fragrances of pollen and different floral parts of male and female flowers of Laurus nobilis. Journal of Agricultural and Food Chemistry, 50(16), 4647-4652. DOI: 10.1021/jf020269x

Free, J. B. 1970. Effect of flower shapes and nectar guides on the behaviour of foraging honeybees. Behaviour, 37, 269-285.

Freitas, L. 2013. Concepts of pollinator performance: is a simple approach necessary to achieve a standardized terminology? Brazilian 
Journal of Botany, 36, 3-8. DOI: 10.1007/s40415013-0005-6

Galen, C., \& Kevan, P. G. 1980. Scent and color, floral polymorphisms and pollination biology in Polemonium viscosum Nutt. American Midland Naturalist, 104(2), 281. DOI: 10.2307/2424867

Gegear, R. J., \& Laverty, T. M. 2001. The effect of variation among floral traits on the flower constancy of pollinators. Cognitive Ecology of Pollination, 7, 1-20.

Giurfa, M., Dafni, A., \& Neal, P. R. 1999. Floral symmetry and its role in plant-pollinator systems. International Journal of Plant Sciences, 160, S41-S50.

Giurfa, M. \& Lehrer, M. 2001. Honeybee vision and floral display: from detection to close-up recognition. Cognitive Ecology of Pollination, 61-82.

Giurfa, M., Nunez, J., Chittka, L., \& Menzel, R. 1995. Colour preferences of flower-naive honeybees. Journal of Comparative Physiology A: Neuroethology, Sensory, Neural, and Behavioral Physiology, 177(3), 247-259.

Giurfa, M., Vorobyev, M., Kevan, P. G., \& Menzel, R. 1996. Detection of coloured stimuli by honeybees: minimum visual angles and receptor specific contrasts. Journal of Comparative Physiology A, 178, 699-709.

Gómez, J. M., Bosch, J., Perfectti, F., Fernández, J. D., Abdelaziz, M., \& Camacho, J. P. M. 2008. Spatial variation in selection on corolla shape in a generalist plant is promoted by the preference patterns of its local pollinators. Proceedings of the Royal Society of London B: Biological Sciences, 275(1648), 2241-2249.

Gottsberger, G., Silberbauer-Gottsberger, I., Seymour, R. S., \& Dötterl, S. 2012. Pollination ecology of Magnolia ovata may explain the overall large flower size of the genus. Flora Morphology, Distribution, Functional Ecology of Plants, 207(2), 107-118. DOI: 10.1016/j. flora.2011.11.003

Goulson, D. 2000. Are insects flower constant because they use search images to find flowers? Oikos, 88(3), 547-552.

Goulson, D., Cruise, J. L., Sparrow, K. R., Harris, A. J., Park, K. J., Tinsley, M. C., \& Gilburn, A. S. 2007. Choosing rewarding flowers; perceptual limitations and innate preferences influence decision making in bumblebees and honeybees.
Behavioral Ecology and Sociobiology, 61(10), 1523-1529.

Goulson, D., Stout, J. C., \& Hawson, S. A. 1997. Can flower constancy in nectaring butterflies be explained by Darwin's interference hypothesis? Oecologia, 112(2), 225-231.

Greggers, U., Koch, G., Schmidt, V., Durr, A., Floriou-Servou, A., Piepenbrock, D., \& Menzel, R. 2013. Reception and learning of electric fields in bees. Proceedings of the Royal Society B: Biological Sciences, 280(1759), 2013052820130528. DOI: $10.1098 / \mathrm{rspb} .2013 .0528$

Greiner, B. 2006. Adaptations for nocturnal vision in insect apposition eyes. International Review of Cytology, 250, 1-46.

Grüter, C., Moore, H., Firmin, N., Helanterä, H., \& Ratnieks, F. L. W. 2011. Flower constancy in honey bee workers (Apis mellifera) depends on ecologically realistic rewards. The Journal of Experimental Biology, 214, 1397-1402. DOI: 10.1242/jeb.050583

Gumbert, A. 2000. Color choices by bumblebees (Bombus terrestris): innate preferences and generalization after learning. Behavioral Ecology and Sociobiology, 48(1), 36-43.

Hanley, M. E., Franco, M., Pichon, S., Darvill, B., \& Goulson, D. 2008. Breeding system, pollinator choice and variation in pollen quality in British herbaceous plants. Functional Ecology, 22(4), 592-598, DOI: 10.1111/j.1365-2435.2008.01415.x

Hansen, D. M., Van der Niet, T., \& Johnson, S. D. 2012. Floral signposts: testing the significance of visual "nectar guides" for pollinator behaviour and plant fitness. Proceedings of The Royal Society B, 279: 634-639.

Hansson, B. S. \& Stensmyr, M. C. 2011. Evolution of insect olfaction. Neuron, 72, 698-711. DOI: 10.1016/j.neuron.2011.11.003

Heinrich, B. 1979. "Majoring" and "Minoring" by Foraging Bumblebees, Bombus vagans: An Experimental Analysis. Ecology, 60(2), 245-255.

Herrera, C. M., Castellanos, M. C. \& Medrano, M. 2006. Geographical context of floral evolution: towards an improved research programme in floral diversification. In: L. D. Harder \& S. C. H. Barrett (Eds.), Ecology and evolution of flowers. pp. 278-294. Oxford, UK: Oxford University Press.

Herrera, C. M., García, I. M., \& Pérez, R. 2008. Invisible floral larcenies: microbial commu- 
nities degrade floral nectar of bumble beepollinated plants. Ecology, 89(9), 2369-2376. DOI: 10.1890/08-0241.1

Hetherington-Rauth, M. C., \& Ramírez, S. R. 2016. Evolution and diversity of floral scent chemistry in the euglossine bee-pollinated orchid genus Gongora. Annals of Botany, 118, 135-148. DOI: 10.1093/aob/mcw072

Hills, H. G., Williams, N. H., \& Dodson, C. H. 1972. Floral Fragrances and Isolating Mechanisms in the Genus Catasetum (Orchidaceae). Biotropica, 4(2), 61. DOI: $10.2307 / 2989728$

Hopkins, M. J. G., Hopkins, H. C. F., \& Sothers, C. A. 2000. Nocturnal pollination of Parkia velutina by Megalopta bees in Amazonia and its possible significance in the evolution of chiropterophily. Journal of Tropical Ecology, 16(5), 733-746.

Howell, A. D., \& Alarcón, R. 2007. Osmia bees (Hymenoptera: Megachilidae) can detect nectar-rewarding flowers using olfactory cues. Animal Behaviour, 74, 199-205. DOI: 10.10 16/j.anbehav.2006.11.012

Ibarra, N. H., \& Giurfa, M. 2003. Discrimination of closed coloured shapes by honeybees requires only contrast to the long wavelength receptor type. Animal Behaviour, 66(5), 903-910.

Ida, T. Y., \& Kudo, G. 2003. Floral color change in Weigela middendorffiana (Caprifoliaceae): reduction of geitonogamous pollination by bumblebees. American Journal of Botany, 90(12), 1751-1757.

Ings, T. C., Raine, N. E., \& Chittka, L. 2009. A population comparison of the strength and persistence of innate colour preference and learning speed in the bumblebee Bombus terrestris. Behavioral Ecology and Sociobiology, 63(8), 1207-1218.

Inouye, D. W. 1980. The effect of proboscis and corolla tube lengths on patterns and rates of flower visitation by bumblebees. Oecologia, (45), 197-201.

Irwin, R. E., Bronstein, J. L., Manson, J. S., \& Richardson, L. 2010. Nectar robbing: ecological and evolutionary perspectives. Annual Review of Ecology, Evolution, and Systematics, 41(1), 271-292. DOI: 10.1146/annurev.ecolsys.110308. 120330

Isidorov, V. A., Zenkevich, I. G., \& Ioffe, B. V. 1985. Volatile organic compounds in the atmosphere of forests. Atmospheric Environment, (1967), 19(1), 1-8. DOI: 10.1016/0004-6981(85)90131-3

Jander, U., \& Jander, R. 2002. Allometry and resolution of bee eyes (Apoidea). Arthropod Structure \& Development, 30(3), 179-193.

Jersáková, J., Johnson, S. D. \& Kindlmann, P. 2006. Mechanisms and evolution of deceptive pollination in orchids. Biological Review, 81, 219-235.

Jersáková, J., Jurgens, A., Smilauer, P., Johnson, S. D. 2012. The evolution of floral mimicry: identifying traits that visually attract pollinators. Functional Ecology, 26, 1381-1389.

Johnson, S. D., Peter, C. I., Nilsson, L. A., \& Ågren, J. 2003. Pollination success in a deceptive orchid is enhanced by co-occurring rewarding magnet plants. Ecology, 84(11), 2919-2927. DOI: 10.18 90/02-0471

Junker, R. R., \& Parachnowitsch, A. L. 2015. Working towards a holistic view on flower traitshow floral scents mediate plant-animal interactions in concert with other floral characters. Journal of the Indian Institute of Science: A Multidisciplinary Reviews Journal, 95(1), 1-13. DOI: $10.1111 /$ evo. 12558

Junker, R. R., \& Blüthgen, N. 2008. Floral scents repel potentially nectar-thieving ants. Evolutionary Ecology Research, 10, 295-308.

Junker, R. R., \& Blüthgen, N. 2010. Floral scents repel facultative flower visitors, but attract obligate ones. Annals of Botany, 105(5), 777782. DOI: $10.1093 / \mathrm{aob} / \mathrm{mcq} 045$

Jürgens, A., Dötterl, S., \& Meve, U. 2006. The chemical nature of fetid floral odours in stapeliads (Apocynaceae, sclepiadoideae, Ceropegieae). New Phytologist, 172(3), 452-468. DOI: $10.1111 /$ j.1469-8137.2006.01845.x

Jürgens, A., Wee, S. L., Shuttleworth, A., \& Johnson, S. D. 2013. Chemical mimicry of insect oviposition sites: A global analysis of convergence in angiosperms. Ecology Letters, 16(9), 1157-1167. DOI: 10.1111/ele.12152

Kaczorowski, R. L., Leonard, A. S., Dornhaus, A., \& Papaj, D. R. 2012. Floral signal complexity as a possible adaptation to environmental variability: a test using nectar-foraging bumblebees, Bombus impatiens. Animal Behaviour, 83(4), 905-913.

Kantsa, A., Raguso, R. A., Dyer, A. G., Sgardelis, S. P., Olesen, J. M., \& Petanidou, T. 2017. 
Community-wide integration of floral colour and scent in a Mediterranean scrubland. Nature Ecology \& Evolution, 1, 1502-1510. DOI: 10.1038/s41559-017-0298-0

Kárpáti, Z., Knaden, M., Reinecke, A., \& Hansson, B. S. 2013. Intraspecific combinations of flower and leaf volatiles act together in attracting hawkmoth pollinators. PLoS ONE, 8(9), e72805. DOI: 10.1371/journal.pone.0072805

Katzenberger, T. D., Lunau, K., \& Junker, R. R. 2013. Salience of multimodal flower cues manipulates initial responses and facilitates learning performance of bumblebees. Behavioral Ecology and Sociobiology, 67, 1587-1599. DOI: $10.1007 /$ s00265-013-1570-1

Kelber, A., Balkenius, A., Warrant, E. J. 2003. Colour vision in diurnal and nocturnal hawkmoths. Integrative and Comparative Biology, 43(4), 1, 571-579. DOI: 10.1093/icb/43. 4.571

Kelber, A., Warrant, E. J., Pfaff, M., Wallén, R., Theobald, J. C., Wcislo, W. T., \& Raguso, R. A. 2005. Light intensity limits foraging activity in nocturnal and crepuscular bees. Behavioral Ecology, 17(1), 63-72. DOI: 10.1093/beheco/ arj001

Kemp, D. J., Herberstein, M. E., Fleishman, L. J., Endler, J. A., Bennett, A. T. D., Dyer, A. G., Hart, N. S., Marshall, J., \& Whiting, M. J. 2015. An integrative framework for the appraisal of coloration in nature. The American Naturalist, 185(6), 705-724. DOI: 10.1086/681021

Kessler, A., Halitschke, R., \& Poveda, K. 2011. Herbivory-mediated pollinator limitation: Negative impacts of induced volatiles on plantpollinator interactions. Ecology, 92(9), 17691780. DOI: 10.1890/10-1945.1

Kessler, D., Gase, K., \& Baldwin, I. T. 2008. Field experiments with transformed plants reveal the sense of floral scents. Science, 321(5893), 12001202.

Kessler, S. C., Tiedeken, E. J., Simcock, K. L., Derveau S., Mitchell, S. S., Stout, J. C., \& Wright, G. A. 2015. Bees prefer foods containing neonicotinoid pesticides. Nature, 521, 74-76. DOI: $10.1038 /$ nature14414

Kevan, P. G., \& Lane, M. A. 1985. Flower petal microtexture is a tactile cue for bees. Proceedings of the National Academy of Sciences, 82(14), 4750-4752. DOI: 10.1073/pnas.
82.14.4750

Kevan, P. G. \& Meredith, A. L. 1985. Proceedings of the National Academy of Sciences, (82), 47504752.

Kirschvink, J. L., \& Gould, J. L. 1981. Biogenic magnetite as a basis for magnetic field detection in animals. Biosystems, 13(3), 181-201. DOI: 10.1016/0303-2647.

Kleineidam, C., Romani, R., Tautz, J., \& Isidoro, N. 2000. Ultrastructure and physiology of the $\mathrm{CO} 2$ sensitive sensillum ampullaceum in the leafcutting ant Atta sexdens. Arthropod Structure \& Development, 29(1), 43-55.

Klinkhamer, P. G. L. \& de Jong, T. J. 1990. Effects of plant size, plant density and sex differential nectar reward on pollinator visitation in the protandrous Echiurn vulgare (Boraginaceae). Oikos, 57, 399405.

Knudsen, J. T. J., Eriksson, R., Gershenzon, J., \& Ståhl, B. 2006. Diversity and distribution of floral scent. The Botanical Review, 72(1), 1-120. DOI: 10.1663/0006-8101(2006)72

Koski, M. H., \& Ashman, T.-L. 2015. Floral pigmentation patterns provide an example of Gloger's rule in plants. Nature Plants, 1(1), 14007. DOI: $10.1038 /$ nplants.2014.7

Koski, M. H., \& Ashman, T.-L. 2016. Macro evolutionary patterns of ultraviolet floral pigmentation explained by geography and associated bioclimatic factors. New Phytologist, 211(2), 708-718. DOI: 10.1111/nph.13921

Kulahci, I. G., Dornhaus, A., \& Papaj, D. R. 2008. Multimodal signals enhance decision making in foraging bumble-bees. Proceedings. Biological Sciences / The Royal Society, 275(1636), 797802. DOI: $10.1098 / \mathrm{rspb} .2007 .1176$

Kunze, J. 2001. The combined effect of color and odor on flower choice behavior of bumblebees in flower mimicry systems. Behavioral Ecology, 12(4), 447-456. DOI: 10.1093/beheco/12.4.447

Land, M. F. 1997. Visual acuity in insects. Annual review of Entomology, 42(1), 147-177.

Land, M. F., \& Nilsson, D. E. 2002. Animal eyes. Oxford: Oxford University Press: p. 271.

Lehrer, M. 1999. Shape perception in the honeybee: symmetry as a global framework. International Journal of Plant Sciences, 160, S51-S65.

Lehrer, M., Horridge, G. A., Zhang, S. W., \& Gadagkar, R. 1995. Shape vision in bees: innate 
preference for flower-like patterns. Philosophical Transactions of the Royal Society B: Biological Sciences, 347(1320), 123-137. DOI: 10.1098/rstb.1995.0017

Leonard, A. S., \& Francis, J. S. 2017. Plant-animal communication: past, present and future. Evolutionary Ecology, 31(2), 143-151. DOI: 10.1007/s10682-017-9884-5

Leonard, A. S., \& Masek, P. 2014. Multisensory integration of colors and scents: insights from bees and flowers. Journal of Comparative Physiology A: Neuroethology, Sensory, Neural, and Behavioral Physiology, 200, 463-474. DOI: 10.1007/s00359-014-0904-4

Leonard, A. S., \& Papaj, D. R. 2011. 'X' marks the spot: The possible benefits of nectar guides to bees and plants. Functional Ecology, 25(6), 1293-1301. DOI: $10.1111 /$ j.1365-2435.2011.0188 5. $\mathrm{x}$

Leonard, A. S., Brent, J., Papaj, D. R., \& Dornhaus, A. 2013. Floral nectar guide patterns discourage nectar robbing by bumble bees. PLoS ONE, 8(2), e55914. DOI: 10.1371/journal.pone.0055914

Leonard, A. S., Dornhaus, A., \& Papaj, D. R. 2011. Forget-me-not: Complex floral displays, intersignal interactions, and pollinator cognition. Current Zoology, 57(2), 215-224.

Leppik, E. E. 1953. The ability of insects to distinguish number. American Naturalist, 87, $229-236$.

Lihoreau, M., \& Raine, N. E. 2013. Bee positive: the importance of electroreception in pollinator cognitive ecology. Frontiers in Psychology, 4, 445. DOI:10.3389/fpsyg.2013.00445.

Loukola, O. J., Perry, C. J., Coscos, L., \& Chittka, L. 2017. Bumblebees show cognitive flexibility by improving on an observed complex behavior. Science, 355(6327), 833-836. DOI: 10.1126/scie nce.aag2360

Lucas-Barbosa, D., Van Loon, J. J. A., \& Dicke, M. 2011. The effects of herbivore-induced plant volatiles on interactions between plants and flower-visiting insects. Phytochemistry, 72(13), 1647-1654. DOI: 10.1016/j.phytochem.2011.03. 013

Lucas-Barbosa, D., Van Loon, J. J. A., Gols, R., van Beek, T. A. \& Dicke, M. 2013. Reproductive escape: annual plant responds to butterfly eggs by accelerating seed production. Functional Ecology, 27, 245-254.
Lunau, K. 1990. Colour saturation triggers innate reactions to flower signals: flower dummy experiments with bumblebees. Journal of Comparative Physiology A: Neuroethology, Sensory, Neural, and Behavioral Physiology, 166(6), 827-834.

Lunau, K. 2000. The ecology and evolution of visual pollen signals. Plant Sytstematics and Evolution, 222, 89-111.

Lunau, K., \& Maier, E. J. 1995. Innate colour preferences of flower visitors. Journal of Comparative Physiology A, 177, 1-19.

Lunau, K., Konzmann, S., Winter, L., Kamphausen, V., \& Ren, Z. X. 2017. Pollen and stamen mimicry: the alpine flora as a case study. Arthropod-Plant Interactions, 3(11), 427-447.

Lunau, K., Papiorek, S., Eltz, T., \& Sazima, M. 2011. Avoidance of achromatic colours by bees provides a private niche for hummingbirds. The Journal of Experimental Biology, 214, 16071612. DOI: $10.1242 /$ jeb.052688

Lunau, K., Wacht, S., \& Chittka, L. 1996. Colour choices of naive bumblebees and their implications for colour perception. Journal of Comparative Physiology A: Neuroethology, Sensory, Neural, and Behavioral Physiology, 178(4), 477-489.

Makino, T. T., \& Ohashi, K. 2016. Honest signals to maintain a long-lasting relationship: floral colour change prevents plant-level avoidance by experienced pollinators. Functional Ecology, 31, 831-837. DOI: 10.1111/1365-2435.12802

Márquez, M. A. 2009. Floral constancy in bees: a revision of theories and a comparison with other pollinators. Revista Colombiana de Entomologia, 35(2), 206-216.

Martin, H. \& Lindauer, M. 1966. Flower petal microtexture is a tactile cue for bees. Zeitschrift für Vergleichende Physiologie, 53, 372-404.

Mathur, G., \& Ram, M. 1978. Significance of petal colour in thrips-pollinated Lantana Camara. Annals of Botany, 42, 1473-1476.

McEwen, J. R. \& Vamosi, J. C. 2010. Floral colour versus phylogeny in structuring subalpine flowering communities. Proceedings of the Royal Society Biological Sciences, 277, 29572965; DOI: 10.1098/rspb.2010.0501

Menzel, R. 1985. Learning in honeybees in an ecological and behavioral context. In: B. Hlldobler \& M. Lindauer (Eds.), Experimental 
Behavioral Ecology. pp. 55-74. Fischer, Stuttgart.

Mesquita-Neto, J. N., Costa, B. K. P., \& Schlindwein, C. 2016. Heteranthery as a solution to the demands for pollen as food and for pollination - Legitimate flower visitors reject flowers without feeding anthers. Plant Biology, 19(6), 942-950. DOI: 10.1111/plb.12609

Michener, C. D. 2000. The bees of the world. Baltimore and London: The John Hopkins University Press: p. 913.

Milet-Pinheiro, P., \& Gerlach, G. 2017. Biology of the Neotropical orchid genus Catasetum: A historical review on floral scent chemistry and pollinators. Perspectives in Plant Ecology, Evolution and Systematics, 27, 23-34. DOI: 10.1016/j.ppees.2017.05.004

Milet-Pinheiro, P., Ayasse, M., \& Dötterl, S. 2015. Visual and olfactory floral cues of Campanula (Campanulaceae) and their significance for host recognition by an oligolectic bee pollinator. Plos One, 10(6), e0128577. DOI: 10.1371/journal. pone. 0128577

Milet-Pinheiro, P., Ayasse, M., Schlindwein, C., Dobson, H. E. M., \& Dötterl, S. 2012. Host location by visual and olfactory floral cues in an oligolectic bee: innate and learned behavior. Behavioral Ecology, 23(3), 531-538. DOI: 10.1093/beheco/arr219

Milet-Pinheiro, P., Herz, K., Dötterl, S., \& Ayasse, M. 2016. Host choice in a bivoltine bee: How sensory constraints shape innate foraging behaviors. BMC Ecology, 16(1), 1-12. DOI: 10.1186/s12898-016-0074-Z

Mitchell, R. J., Flanagan, R. J., Brown, B. J., Waser, N. M., \& Karron, J. D. 2009b. New frontiers in competition for pollination. Annals of Botany, 103(9), 1403-1413. DOI: 10.1093/aob/mcp062

Mitchell, R. J., Irwin, R. E., Flanagan, R. J., \& Karron, J. D. 2009a. Ecology and evolution of plant-pollinator interactions. Annals of Botany, 103(9), 1355-63. DOI: 10.1093/aob/mcp122

Mitchell, R. J, Karron, J. D, Holmquist, K. G, \& Bell, J. M. 2004. The influence of Mimulus ringens floral display size on pollinator visitation patterns. Functional Ecology, 18, 116-124.

Mitko, L., Weber, M. G., Ramirez, S. R., Hedenström, E., Wcislo, W. T., \& Eltz, T. 2016. Olfactory specialization for perfume collection in male orchid bees. The Journal of
Experimental Biology, 219(10), 1467-1475. DOI: 10.1242/jeb.136754

Mizunami, M. 1995. Functional diversity of neural organization in insect ocellar systems. Vision Research, 35(4), 443-452.

Møller, A. P. \& Thornhill, R. 1998. Bilateral symmetry and sexual selection: a meta-analysis. The American Naturalist, 151, 174-192.

Møller, A. P. 1990. Fluctuating asymmetry in male sexual ornaments may reliably reveal male quality. Animal Behavior, 40, 1185-1187.

Møller, A. P. 2000. Developmental stability and pollination. Oecologia, 123, 149-157.

Møller, A. P \& Eriksson, M. 1995. Pollinator preference for symmetrical flowers and sexual selection in plants. Oikos, 73,15-22.

Muth, F., Francis, J. S., \& Leonard, A. S. 2016. Bees use the taste of pollen to determine which flowers to visit. Biology Letters, 12(7), 20160356. DOI: $10.1098 /$ rsbl.2016.0356

Muth. F., Cooper, T. R, Bonilla, R. F, \& Leonard, A. S. 2018. A novel protocol for studying bee cognition in the wild. Methods in Ecology and Evolution, 9, 78-87. DOI: 10.1111/2041-210 X.12852

Nathans, J. 1999. The evolution and physiology of human color vision: insights from molecular genetic studies of visual pigments. Neuron, 24(2), 299-312.

Naug, D. \& Arathi, H. S. 2007. Receiver bias for exaggerated signals in honeybees and its implications for the evolution of floral displays. Biology Letters, 3, 635-637.

Ne'eman, G., \& Ne'eman, R. 2017. Factors determining visual detection distance to real flowers by Bumble bees. Journal of Pollination Ecology, 20(1), 1-12.

Neal, P. R., Dafni, A., \& Giurfa, M. 1998. Floral symmetry and its role in plant-pollinator sytems: terminology, distribution, and hypotheses. Annual Review of Ecology and Systematics, 29(1), 345-373. DOI: 10.1146/annu rev.ecolsys.29.1.345

Nepi, M., von Aderkas, P., Wagner, R., Mugnaini, S., Coulter, A., \& Pacini, E. 2009. Nectar and pollination drops: how different are they? Annals of Botany, 104(2), 205-219. DOI: 10.10 93/aob/mcp124.

Nishihara, M., \& Nakatsuka, T. 2011. Genetic engineering of flavonoid pigments to modify 
flower color in floricultural plants. Biotechnology Letters, 33(3), 433-441.

Nishihara, M., Nakatuska, T. 2010. Genetic engineering of novel flower colors in floricultural plants: Recent advances via transgenic approaches. In: S. M. Jain \& S. J. Ochatt (Eds.), Protocols for in vitro propagation of ornamental plants. pp. 325-347. Humana Press, Totowa, New Jersey.

Nunes, C. E., Gerlach, G., Bandeira, K. D., GobboNeto, L., Pansarin, E. R., \& Sazima, M. 2016. Two orchids, one scent? Floral volatiles of Catasetum cernuum and Gongora bufonia suggest convergent evolution to a unique pollination niche. Flora (Jena), 232, 207-216. DOI: 10.1016/j.flora.2016.11.016

Ohashi, K. \& Yahara, T. 2001. Behavioral responses of pollinators to variation in floral display size and their influences on the evolution of floral traits. In: L. Chittka \& J. D. Thomson (Eds.), Cognitive Ecology of Pollination: Animal Behaviour and Floral Evolution. pp. 274-296. Cambridge University Press, New York.

Ohashi, K. \& Yahara, T. 2002. Visit larger displays but probe proportionally fewer flowers: counterintuitive behaviour of nectar-collecting bumblebees achieves an ideal free distribution. Functional Ecology, 16, 492-503.

Ohashi, K., Makino, T. T., \& Arikawa, K. 2015. Floral colour change in the eyes of pollinators: testing possible constraints and correlated evolution. Functional Ecology, 29, 1144-1155. DOI: $10.1111 / 1365-2435.12420$

Ollerton, J., Grace, J., \& Smith, K. 2007. Pollinator behaviour and adaptative floral colour change in Anthophora alluadii (Hymenoptera: Apidae) and Erysimun scoparium (Brasicaceae) on Tenerife. Entomologia Generalis, 29(2/4), 253268.

Onagbola, E. O., Meyer, W. L., Boina, D. R., \& Stelinski, L. L. 2008. Morphological characterization of the antennal sensilla of the Asian citrus psyllid, Diaphorina citri Kuwayama (Hemiptera: Psyllidae), with reference to their probable functions. Micron, 39(8), 1184-1191. DOI: 10.1016/j.micron.2008.05.002

Ozaki, M. 2005. Ant nestmate and non-nestmate discrimination by a chemosensory sensillum. Science, 309(5732), 311-314. DOI: 10.1126/sci ence.1105244
Pansarin, L. M., Pansarin, E. R., \& Sazima, M. 2014. Osmophore structure and phylogeny of Cirrhaea (Orchidaceae, Stanhopeinae). Botanical Journal of the Linnean Society, 176(3), 369-383. DOI: $10.1111 /$ boj.12206

Parachnowitsch, A. L., Raguso, R. A., \& Kessler, A. 2012. Phenotypic selection to increase floral scent emission, but not flower size or colour in bee-pollinated Penstemon digitalis. New Phytologist, 195(3), 667-675. DOI: 10.1111/j.14698137.2012.04188.x

Pareja, M., Qvarfordt, E., Webster, B., Mayon, P., Pickett, J., Birkett, M., \& Glinwood, R. 2012. Herbivory by a phloem-feeding insect inhibits floral volatile production. PLoS ONE, 7(2). DOI: 10.1371/journal.pone.0031971

Paulino-Neto, H. F. 2014. Polinização e biologia reprodutiva de Araticum-liso (Annona coriacea Mart.: Annonaceae) em uma área de cerrado paulista: implicações para fruticultura. Revista Brasileira de Fruticultura, 36, 132-140.

Peitsch, D., Fietz, A., Hertel, H., de Souza, J., Ventura, D. F., \& Menzel, R. 1992. The spectral input systems of hymenopteran insects and their receptor-based colour vision. Journal of Comparative Physiology A Sensory, Neural, and Behavioral Physiology, 170(1), 23-40.

Pélabon, C., Thöne, P., Hansen, T. F., \& Armbruster, W. S. 2012. Signal honesty and cost of pollinator rewards in Dalechampia scandens (Euphorbiaceae). Annals of botany, 109(7), 1331-1340.

Pierik, R., Ballaré, C. L., \& Dicke, M. 2014. Ecology of plant volatiles: taking a plant community perspective. Plant, Cell \& Environment, 37(8), 1845-1853. DOI: $10.1111 /$ pce. 12330

Pinheiro, M., Gaglianone, M. C., Nunes, C. E. P., Sigrist, M. R., Santos, I. A. 2014. Polinização por abelhas. In: A. R. Rech, K. Agostini, P. E. A. M. De Oliveira, \& I. C. Machado (Eds.), Biologia da Polinização. pp. 417-438. Rio de Janeiro: Editora Projeto Cultural.

Potts, S. G., Vulliamy, B., Dafni, A., Ne'eman, G., \& Willmer, P. 2003. Linking bees and flowers: how do floral communities structure pollinator communities? Ecology, 84(10), 2628-2642. DOI: 10.1890/02-0136

Praagh, J. V., Ribi, W., Wehrhahn, C., \& Wittmann, D. 1980. Drone bees fixate the queen with the dorsal frontal part of their compound eyes. 
Journal of Comparative Physiology A: Neuroethology, Sensory, Neural, and Behavioral Physiology, 136(3), 263-266.

Praz, C. J., Muller, \& Dorn, S. 2008. Host recognition in a pollen-specialist bee: evidence for a genetic basis. Apidologie, 39(5), 547-557. DOI: Doi 10.1051/Apido:2008034

Raguso, R. A. 2004. Flowers as sensory billboards: progress towards an integrated understanding of floral advertisement. Current Opinion in Plant Biology, 7, 434-440. DOI: 10.1016/j.pbi. 2004.05.010

Raguso, R. A. 2004. Why Are Some Floral Nectars Scented? Ecology, 85(6), 1486-1494.

Raguso, R. A. 2008. The "invisible hand" of floral chemistry. Science, 321(5893), 11631164. DOI: $10.1126 /$ science. 1163570

Raguso, R. A. 2008. Wake Up and smell the roses: the ecology and evolution of floral scent. The Annual Review of Ecology, Evolution, and Systematics, 39, 549-569. DOI: 10.1146/annu rev.ecolsys.38.091206.095601

Raguso, R. A., \& Willis, M. A. 2002. Synergy between visual and olfactory cues in nectar feeding by wild hawkmoths, Manduca sexta. Animal Behaviour, 69(2), 407-418. DOI: 10. 1016/j.anbehav.2004.04.015

Raguso, R. A., Levin, R. A., Foose, S. E., Holmberg, M. W., \& McDade, L. A. 2003. Fragrance chemistry, nocturnal rhythms and pollination "syndromes" in Nicotiana. Phytochemistry, 63(3), 265-284. DOI: 10.1016/S0031-9422(03)00 113-4

Raine, N. E, \& Gill, R. J. 2015. Tasteless pesticides affect bees in the field. Nature 521, 38-39. DOI: 10.1038 / nature 14391

Raine, N. E., \& Chittka, L. 2007. The adaptive significance of sensory bias in a foraging context: floral colour preferences in the bumblebee Bombus terrestris. PLoS ONE, 2(6), e556. DOI: 10.1371/journal.pone.0000556

Raine, N. E., Ings, T. C., Dornhaus, A., Saleh, N., \& Chittka, L. 2006. Adaptation, genetic drift, pleiotropy, and history in the evolution of bee foraging behavior. Advances in the Study of Behavior, 36, 305-354.

Ram, H. M., \& Mathur, G. 1984. Flower colour changes in Lantana camara. Journal of Experimental Botany, 35(11), 1656-1662.

Ramírez, S. R., Eltz, T., Fritzsch, F., Pemberton, R.,
Pringle, E. G., \& Tsutsui, N. D. 2010. Intraspecific geographic variation of fragrances acquired by orchid bees in native and introduced populations. Journal of Chemical Ecology, 36(8), 873-884. DOI: 10.1007/s10886010-9821-3

Ravaiano, S. V., Ferreira, R. de P., Campos, L. A. de O., \& Martins, G. F. 2014. The antennal sensilla of Melipona quadrifasciata (Hymenoptera: Apidae: Meliponini): a study of different sexes and castes. Naturwissenschaften, 101(8), 603611. DOI: $10.1007 /$ s00114-014-1184-0

Rech, A. R., Agostini, K., Oliveira, P. E., \& Machado, I. C. 2014. Biologia da polinização. Editora Projeto Cultural, Rio de Janeiro. p. 524.

Reinhard, J., Sinclair, M., Srinivasan, M. V., \& Claudianos, C. 2010. Honeybees learn odour mixtures via a selection of key odorants. PLoS ONE, 5(2), 1-14. DOI: 10.1371/journal.pone.00 09110

Rering, C. C., Beck, J. J., Hall, G. W., McCartney, M. M., \& Vannette, R. L. 2018. Nectar-inhabiting microorganisms influence nectar volatile composition and attractiveness to a generalist pollinator. New Phytologist, 220(3), 750-759 DOI: $10.1111 / \mathrm{nph} .14809$

Reverté, S., Retana, J., Gómez, J. M., \& Bosch, J. 2016. Pollinators show flower colour preferences but flowers with similar colours do not attract similar pollinators. Annals of Botany, 118(2), 249-257. DOI: 10.1093/aob/mcw103

Riffell, J. A. 2011. The neuroecology of a pollinator's buffet: Olfactory preferences and learning in insect pollinators. Integrative and Comparative Biology, 51(5), 781-793. DOI: 10.1093/icb/icr094

Riffell, J. A., Shlizerman, E., Sanders, E., Abrell, L., Medina, B., Hinterwirth, A. J., \& Kutz, J. N. 2014. Flower discrimination by pollinators in a dynamic chemical environment. Science, 344 (6191), 1515-1518.

Rodríguez, I., Gumbert, A., de Ibarra, N. H., Kunze, J., \& Giurfa, M. 2004. Symmetry is in the eye of the 'beeholder': innate preference for bilateral symmetry in flower-naïve bumblebees. Naturwissenschaften, 91(8), 374-377.

Rodríguez-Gironés, M. A., \& Santamaría, L. 2004. Why are so many bird flowers red? PLOS Biology, 2(10), e350.

Rodríguez-Gironés, M. A., Trillo, A., \& Corcobado, 
G. 2013. Long term effects of aversive reinforcement on colour discrimination learning in free-flying bumblebees. PLoS ONE, 8(8), e71551. DOI: 10.1371/journal.pone.00715 51

Rowe, C. 1999. Receiver psychology and the evolution of multicomponent signals. Animal Behaviour, 58, 921-931.

Rowe, C. 2013. Receiver psychology: a receiver's perspective. Animal Behaviour, 85(3), 517-523. DOI: 10.1016/j.anbehav.2013.01.004

Rusch, C., Broadhead, G. T., Raguso, R. A., \& Riffell, J. A. 2016. Olfaction in context-sources of nuance in plant-pollinator communication. Current Opinion in Insect Science, 15, 53-60. DOI: 10.1016/j.cois.2016.03.007

Sánchez, D., Nieh, J. C., \& Vandame, R. 2011. Visual and chemical cues provide redundant information in the multimodal recruitment system of the stingless bee Scaptotrigona mexicana (Apidae, Meliponini). Insectes Sociaux, 58, 575-579. DOI: 10.1007/s00040-0110181-y

Sargent, R. D. 2004. Floral symmetry affects speciation rates in angiosperms. Proceedings of the Royal Society B: Biological Sciences, 271(1539), 603-608. DOI: 10.1098/rspb.2003.26 44

Sarmento, R. A., de Lemos, F., Dias, C. R., Pallini, A., \& Venzon, M. 2008. Infoquímicos induzidos por herbivoria mediando a comunicação entre plantas de tomate e o predador Cycloneda sanguinea (Coleoptera: Coccinellidae). Ceres, 55(5), 439-444.

Sato, K., \& Touhara, K. 2008. Insect Olfaction: Receptors, Signal Transduction, and Behavior. In: S. Korsching \& W. Meyerhof (Eds.), Chemosensory Systems in Mammals, Fishes, and Insects. pp. 203-220. Springer Berlin Heidelberg, Berlin, Heidelberg. DOI: 10.1007/ 978-3-540-69919-4

Sazima, M., Buzato, S., \& Sazima, I. 1999. Batpollinated flower assemblages and bat visitors at two Atlantic forest sites in Brazil. Annals of Botany, 83(6), 705-712.

Sazima, M., Vogel, S., Cocucci, A., \& Hausner, G. 1993. The perfume flowers of Cyphomandra (Solanaceae): Pollination by euglossine bees, bellows mechanism, osmophores, and volatiles. Plant Systematics and Evolution, 187(1), 51-88.
Schaefer, H. M. \& Ruxton, G. D. 2010. Deception in plants: mimicry or perceptual exploitation? Trends in Ecology \& Evolution, 24, 676-684.

Scheiner, R, \& Amdam, G. V. 2009. Impaired tactile learning is related to social role in honeybees. The Journal of Experimental Biology, 212(7), 994-1002. DOI: 10.1242/jeb.021188.

Scheiner, R., Kuritz-Kaiser, A., Menzel, R., \& Erber, J. 2005. Sensory responsiveness and the effects of equal subjective rewards on tactile learning and memory of honeybees. Learning \& Memory, 12(6), 626-635. DOI: 10.1101/lm.98105

Scheiner, R., Barnert, M., \& Erber, J. 2003. Variation in water and sucrose responsiveness during the foraging season affects proboscis extension learning in honey bees. Apidologie, 34, 67-72. DOI: 10.1051/apido:2002050

Scheiner, R., Erber, J., \& Page, R. E. 1999. Tactile learning and the individual evaluation of the reward in honey bees (Apis mellifera L.). Journal of Comparative Physiology A 185, 1-10. DOI: $10.1007 / \mathrm{s} 003590050360$

Scheiner, R., Page, R. E., \& Erber, J. 2001a. Responsiveness to sucrose affects tactile and olfactory learning in preforaging honey bees of two genetic strains. Behavioural Brain Research, 120, 67-73. DOI: 10.1016/S0166-4328(00)003594

Scheiner, R., Page, R. E., \& Erber, J. 2001b. The effects of genotype, foraging role and sucrose perception on the tactile learning performance of honey bees (Apis mellifera L.). Neurobiology of Learning and Memory, 76, 138-150. DOI: 10.1006/nlme.2000.3996

Schiestl, F. P. 2005. On the success of a swindle: pollination by deception in orchids. Naturwissenschaften, 92(6), 255-264. DOI: 10.1007/s0011 4-005-0636-y

Schiestl, F. P. 2010. Pollination: Sexual mimicry abounds. Current Biology, 20(23), R1020-R1022. DOI: $10.1016 /$ j.cub.2010.10.019

Schiestl, F. P. 2015. Ecology and evolution of floral volatile- mediated information transfer in plants. New Phytologist, 206, 571-577. DOI: $10.1111 /$ nph. 13243

Schiestl, F. P., \& Ayasse, M. 2002. Do changes in floral odor cause speciation in sexually deceptive orchids? Plant Systematics and Evolution, 234(1), 111-119.

Schiestl, F. P., \& Roubik, D. W. 2003. Odor 
Compound Detection in Male Euglossine Bees. Journal of Chemical Ecology, 29(1), 253-257. DOI: 10.1023/A:1021932131526

Schiestl, F. P., \& Schlüter, P. M. 2009. Floral isolation, specialized pollination, and pollinator behavior in orchids. Annual Review of Entomology, 54, 425-46. DOI: 10.1146/annurev. ento.54.110807.090603

Schiestl, F. P., Ayasse, M., Paulus, H. F., Löfstedt, C., Hansson, B. S., Ibarra, F., \& Francke, W. 1999. Orchid pollination by sexual swindle. Nature, 399(6735), 421-421.

Schiestl, F. P., Johnson, S. D., \& Raguso, R. A. 2010. Floral evolution as a figment of the imagination of pollinators. Trends in Ecology and Evolution, 25(7), 382. DOI: 10.1016/j.tree.2010.03.004

Schiestl, F. P., Kirk, H., Bigler, L., Cozzolino, S., \& Desurmont, G. A. 2014. Herbivory and floral signaling: Phenotypic plasticity and tradeoffs between reproduction and indirect defense. New Phytologist, 203(1), 257-266. DOI: 10.11 $11 /$ nph. 12783

Schiestl, F. P., Peakall, R., Mant, J. G., Ibarra, F., Schulz, C., Franke, S., \& Francke, W. 2003. The chemistry of sexual deception in an orchidwasp pollination system. Science, 302(5644), 437-438.

Schlindwein, C., Westerkamp, C., Carvalho, A. T., \& Milet-pinheiro, P. 2014. Visual signalling of nectar-offering flowers and specific morphological traits favour robust bee pollinators in the mass-flowering tree Handroanthus impetiginosus (Bignoniaceae). Botanical Journal of the Linnean Society, 176, 396-407.

Shivanna, K. R. 2014. Biotic Pollination. In: A. Maciel-Silva \& C. K. Porto (Eds.), Reproductive Biology of Plants. pp. 218-267. CRC Press. DOI: $10.1201 / \mathrm{b} 16535-12$

Singaravelan, N., Inbar, M., Ne'eman, G., Distl, M., Wink, M., \& Izhaki, I. 2006. The effects of nectar-nicotine on colony fitness of caged honeybees. Journal of Chemical Ecology, 32(1), 49-59.

Singaravelan, N., Nee'man, G., Inbar, M., \& Izhaki, I. 2005. Feeding responses of free-flying honeybees to secondary compounds mimicking floral nectars. Journal of Chemical Ecology, 31(12), 2791-2804. DOI:10.1007/s10886-006-9 350-2

Skorupski, P., \& Chittka, L. 2006. Animal cognition:
An insect's sense of time? Current Biology, 16, R851-R853.

Snyder, A. W. 1977. Acuity of compound eyes: physical limitations and design. Journal of Comparative Physiology A: Neuroethology, Sensory, Neural, and Behavioral Physiology, 116(2), 161-182.

Soares, N. C., \& Morellato, L. P. C. 2017. Crepuscular pollination and reproductive ecology of Trembleya laniflora (Melastomataceae), an endemic species in mountain rupestrian grasslands. Flora, 238, 138-147. DOI: 10.1016/j.flora.2016.12.005

Somanathan, H., Borges, R. M., Warrant, E. J., \& Kelber, A. 2008. Nocturnal bees learn landmark colours in starlight. Current Biology, 18(21), R996-R997.

Spaethe, J., Brockmann, A., Halbig, C., \& Tautz, J. 2007. Size determines antennal sensitivity and behavioral threshold to odors in bumblebee workers. Naturwissenschaften, 94(9), 733-739. DOI: 10.1007/s00114-007-0251-1

Spaethe, J., Tautz, J., \& Chittka, L. 2001. Visual constraints in foraging bumblebees: flower size and color affect search time and flight behavior. Proceedings of the National Academy of Sciences of the United States of America, 98(7), 3898-903. DOI: 10.1073/pnas.071053098

Sprengel, C. K. 1793. Das entdeckte Geheimnis der Natur im Bau und in der Befruchtung der Blumen. Berlin: Vieweg: p. 443.

Srinivasan, M. V., \& Lehrer, M. 1984. Temporal acuity of honeybee vision: Behavioural studies using moving stimuli. Journal of Comparative Physiology A, 155, 297-312.

Stanley, D. A., Otieno, M., Steijven, K., Berlin, E. S., Piironen, T., Willmer, P. Nuttman, C. V. 2016. Pollination ecology of Desmodium setigerum (Fabaceae) in Uganda; do big bees do it better? Journal of Pollination Ecology, 19(7), 43-49.

Stebbins, G. L. 1951. Natural selection and differentiation of Angiosperm families. Evolution, 5, 299-324.

Stebbins, G. L. 1974. Flowering Plants- Evolution Above the Species Level. London: Edward Arnold. p. 399.

Steinbrecht, R. A. 1996. Structure and function of insect olfactory sensilla. Ciba Foundation Symposium, 200, 158-174. DOI: 10.1002/9780 470514948.ch13 
Stengl, M., Ziegelberger, G., Boekhoff, I., \& Krieger, J. 1999. Perireceptor Events and Transduction Mechanisms in Insect Olfaction. In: B. S. Hansson (Ed.), Insect Olfaction. pp. 49-66. Springer Berlin Heidelberg, Berlin, Heidelberg.

Stephenson, A. G. 1981. Flower and fruit abortion: proximate causes and ultimate functions. Annual Review of Ecology and Systematics, 12, 253-279.

Streinzer, M., Paulus, H. F., \& Spaethe, J. 2009. Floral colour signal increases short-range detectability of a sexually deceptive orchid to its bee pollinator. Journal of Experimental Biology, 212(9), 1365-1370.

Sutcliffe, J. F. 1994. Sensory bases of attractancy: morphology of mosquito olfactory sensilla-a review. Journal of the American Mosquito Control Association, 10(2), 309-315.

Sutton, G. P., Clarke, D., Morley, E. L., \& Robert, D. 2016. Mechanosensory hairs in bumblebees (Bombus terrestris) detect weak electric fields. Proceedings of the National Academy of Sciences, 201601624. DOI: 10.1073/pnas.16016 24113

Suzuki, M. F., \& Ohashi, K. 2014. How does a floral colour-changing species differ from its noncolour-changing congener? -a comparison of trait combinations and their effects on pollination. Functional Ecology, 28(3), 549-560.

Tanaka, Y. \& Brugliera, F. 2006. Flower colour. In: C. Ainsworth (Ed.), Flowering an its manipulation. pp. 201-239. Ashford, Blackwell Publishing.

Telles, F. J., Corcobado, G., Trillo, A., \& RodríguezGironés, M. A. 2017. Multimodal cues provide redundant information for bumblebees when the stimulus is visually salient, but facilitate red target detection in a naturalistic background. PLoS ONE, 12(9). DOI: 10.1371/journal.pone.01 84760

Thomson, J. D., Draguleasa, M. A., \& Tan, M. G. 2015. Flowers with caffeinated nectar receive more pollination. Arthropod-Plant Interactions, 9(1), 1-7. DOI: 10.1007/s11829-014-9350-z.

Vaknin, Y., Gan-Mor, S., Bechar, A., Ronen, B., Eisikowitch, D. 2000. The role of electrostatic forces in pollination. Plant Systematics and Evolution, 222, 133-142.

Vidal, W. N. \& Vidal, M. R. R. 2000. Botânica: organografia. Viçosa: UFV. p. 124.
Vogel, S. 1963. Duftdrüsen im Dienste der Bestäubung: über Bau und Funktion der Osmophoren. Akademie der Wissenschaften und der Literatur. p. 165.

Vorobyev, M., Gumbert, A., Kunze, J., Giurfa, M., \& Menzel, R. 1997. Flowers through insect eyes. Israel Journal of Plant Sciences, 45(2-3), 93-101.

Wang, R., Xu, S., Liu, X., Zhang, Y., Wang, J., \& Zhang, Z. 2014. Thermogenesis, flowering and the association with variation in floral odour attractants in Magnolia sprengeri (Magnoliaceae). PLoS ONE, 9(6), e99356. DOI: 10.13 71/journal.pone.0099356

Wang, Y., Li, D., Liu, Y., Li, X.-J., Cheng, W.-N., \& Zhu-Salzman, K. 2016. Morphology, ultrastructure and possible functions of antennal sensilla of Sitodiplosis mosellana Géhin (Diptera: Cecidomyiidae). Journal of Insect Science, 16(1), 93. DOI: 10.1093/jisesa/iew080

Warnke, U. 1976. Effects of electric charges on honeybees. Bee World, 57(2), 50-56. DOI: 10.1080/0005772X.1976.11097592

Warrant, E. J. 2008. Seeing in the dark: vision and visual behaviour in nocturnal bees and wasps. Journal of Experimental Biology, 211(11), 17371746. DOI: $10.1242 /$ jeb.015396

Warrant, E. J., Kelber, A., Gislén, A., Greiner, B., Ribi, W., \& Wcislo, W. T. 2004. Nocturnal vision and landmark orientation in a tropical halictid bee. Current Biology, 14(15), 1309-1318.

Waser, N. M. 1978. Interspecific pollen transfer and competition between co-occurring plant species. Oecologia, 36(2), 223-236.

Waser, N. M. 1986. Flower constancy: definition, cause, and measurement. The American Naturalist, 127(5), 593-603. DOI: 10.1086/28 4507

Waser, N. M., \& Ollerton, J. 2006. Plant-pollinator interactions: from specialization to generalization. Chicago: University of Chicago Press: $p$. 445.

Wcislo, W. T., Arneson, L., Roesch, K., Gonzalez, V., Smith, A., \& Fernández, H. 2004. The evolution of nocturnal behaviour in sweat bees, Megalopta genalis and M. ecuadoria (Hymenoptera: Halictidae): An escape from competitors and enemies? Biological Journal of the Linnean Society, 83(3), 377-387. DOI: 10.1111/j.1095-8312.2004.00399.x

Weiss, M. R. 1995. Floral color change: a wide 
spread functional convergence. American Journal of Botany, 82, 167-186. DOI: $10.2307 / 2445525$

Weiss, M. R., \& Lamont, B. B. 1997. Floral color change and insect pollination: a dynamic relationship. Israel Journal of Plant Sciences, 45(2-3), 185-199. DOI: 10.1080/07929978.1997. 10676683

Westerkamp, C., \& Claßen-Bockhoff, R. 2007. Bilabiate flowers: the ultimate response to bees? Annals of Botany, 100(2), 361-374.

Whitehead, M. R., \& Peakall, R. 2009. Integrating floral scent, pollination ecology and population genetics. Functional Ecology, 23(5), 863-874. DOI: $10.1111 /$ j.1365-2435.2009.01620.x

Whitney, H. M., Chittka, L., Bruce, T. J. A., \& Glover, B. J. 2009. Conical epidermal cells allow bees to grip flowers and increase foraging efficiency. Current Biology, 19(11), 948-953. DOI: 10.1016/j.cub.2009.04.051

Whitten, W. M., Young, A. M., \& Williams, N. H. 1989. Function of glandular secretions in fragrance collection by male euglossine bees (Apidae: Euglossini). Journal of Chemistry Ecology, 15, 1285-1295.

Willmer, P. 2011. Pollination and floral ecology. Princeton University Press. p. 778.

Willmer, P. G., \& Stone, G. N. 1997. How aggressive ant guards assist in seed set in Acacia flowers. Nature, 388, 165-167. DOI: 10.1038/40610

Willmer, P. G., Nuttman, C. V., Raine, N. E., Stone, G. N., Pattrick, J. G., Henson, K., \& Knudsen, J. T. 2009. Floral volatiles controlling ant behaviour. Functional Ecology, 23(5), 888-900. DOI: 10.1111/j.1365-2435.2009.01632.x

Wilson, P., \& Stine, M. 1996. Floral constancy in bumble bees: handling efficiency or perceptual conditioning? Oecologia, 106(4), 493-499. DOI: 10.1007/BF00329707

Wolowski, M., Carvalheiro, L. G.; \& Freitas, L. 2017. Influence of plant-pollinator interactions on the assembly of plant and hummingbird communities. Journal of Ecology, 105(2), 332-344.
Wright, G. A., Baker, D. D., Palmer, M. J., Stabler, D., Mustard, J. A., Power, E. F.\& Stevenson, P. C. 2013. Caffeine in floral nectar enhances a pollinator's memory of reward. Science, 339(6124), 1202-1204, DOI: 10.1126/science.12 28806

Yan, J., Wang, G., Sui, Y., Wang, M., \& Zhang, L. 2016. Pollinator responses to floral colour change, nectar, and scent promote reproductive fitness in Quisqualis indica (Combretaceae). Scientific Reports, 6, 24408. DOI: 10.1038/ srep24408

Yong, E. 2013. Bees can sense the electric fields of flowers. Phenomena: a science salon. National Geographic. Disponível em: http://phenomena. nationalgeographic.com/2013/02/21/bees-cansense-the-electric-fields-of-flowers/. Acesso em novembro de 2017.

Zakon, H. H. 2016. Electric fields of flowers stimulate the sensory hairs of bumblebees. Proceedings of the National Academy of Sciences, 113(26), 201607426. DOI: 10.1073/ pnas. 1607426113

Zhang, W.; Kramer, E. M. \& Davis, C. C. 2016. Differential expression of $\mathrm{CYC} 2$ genes and the elaboration of floral morphologies in hiptage, an Old World genus of Malpighiaceae. International Journal of Plant Sciences, 177(7), 551558. DOI: $10.1086 / 687225$

Zimmermann, Y., Ramírez, S. R., \& Eltz, T. 2009. Chemical niche differentiation among sympatric species of orchid bees. Ecology, 90(11), 2994-3008.

Submetido em: 14/07/2017 Aceito em: 22/08/2018

Editores Associados: Marina Wolowski \& Vinícius L.G. Brito 